\title{
Cross-waves excited by distributed forcing in the gravity-capillary regime
}

\author{
I. Tinao, J. Porter, A. Laverón-Simavilla, and J. Fernández \\ E.T.S.I. Aeronáuticos, Universidad Politécnica de Madrid, 28040 Madrid, Spain
}

(Received 5 June 2013; accepted 4 February 2014; published online 25 February 2014)

\begin{abstract}
Cross-wave descriptions based on a boundary forced nonlinear Schrodinger equation, which have been widely used since Jones [J. Fluid Mech. 138, 53-74 (1984)], rely on the assumption that modulations occur on a slow lengthscale compared with the extent of the forcing. This assumption does not hold for recent higher frequency (large aspect ratio) experiments. We extend the established theory of modulated cross-waves in horizontally vibrated containers by including surface tension and, most importantly, a spatially extended forcing term. The resulting amplitude equations provide predictions for onset values, spatial profiles, and temporal modulations that are compared with previous theory and with experimental measurements. The appearance of temporally modulated solutions, confirmed experimentally, is interpreted as the result of weak symmetry-breaking related to the interaction of waves generated at opposite ends. 12014 AIP Publishing LLC. [http://dx.doi.org/10.1063/1.4865949]
\end{abstract}

\section{INTRODUCTION}

Subharmonic cross-waves commonly arise in wavemaker experiments when sufficient forcing is applied. So-called because of their crosswise orientation with respect to the applied oscillation and the initial harmonic wave field, they were first described by Faraday, in an experiment utilizing a partially submerged vibrating plate, as forming a series of apparently permanent ridges projecting outward "like the teeth of a coarse comb". ${ }^{1}$ The instability is typically vigorous, with the subharmonic waves quickly overwhelming the initial harmonic wave field, and widespread, occurring not only with plane wavemakers but with virtually any vibrating solid object or boundary, partially or entirely submerged. ${ }^{1-7}$ The parametric nature of the instability, and its origin in the periodic displacement of fluid by a moving boundary, attests to the similarity between cross-waves and the more-studied case of vertically forced subharmonic waves or, simply, "Faraday waves." The localized nature of the forcing mechanism, however, makes them distinct. In a vertically forced Faraday wave experiment, the excitation is homogeneous and the underlying state is trivial (motionless in a comoving frame). The subharmonic instability is the primary one and gives rise to extended patterns, the selection among which is strongly influenced by both temporal and spatial resonances. ${ }^{8-12}$ In a cross-wave experiment, on the other hand, the presence of a directly forced harmonic wave field complicates theoretical analysis of the parametric instability, as does the spatial inhomogeneity of the forcing,

A proper theoretical understanding of cross-waves began with Garrett, ${ }^{13}$ who showed that the onset of instability could be described by Mathieu's equation and is thus parametric in nature. $\mathrm{He}$ found good agreement between his theory and the experiments of Lin and Howard, ${ }^{14}$ particularly with respect to the rightward shift of the frequency-amplitude curves. Further progress was made, following Jones, ${ }^{15}$ by considering the limit of weak damping and weak localized forcing, where the extent of the evanescent field near the wavemaker is much less that the modulation lengthscale of the subharmonic waves. A multiple-scale technique then accounts for the forcing via the boundary condition for an amplitude equation that describes the slow spatial and temporal evolution of the complex cross-wave amplitude $A(x, t)$. The nonlinear Schrödinger (NLS) equation derived by Jones ${ }^{15}$ was subsequently extended by others to include viscous effects ${ }^{16-18}$ and mode interactions. ${ }^{19}$ These 
theoretical models were compared successfully to experiment ${ }^{18,20-22}$ and capture many features of cross-wave behaviour.

Despite their success, the NLS models are clearly not applicable to all cross-wave experiments. They are limited by the assumptions underlying their derivation including the neglect of surface tension and the assumption of very localized forcing. The latter assumption, in particular, is quite restrictive. ${ }^{23}$ It requires either a shallow wavemaker, which would need to be driven at high amplitude to excite cross-waves, or a small dimensionless damping parameter (low viscosity and/or low frequency), to allow the cross-wave field to extend far into the underforced region away from the wavemaker. For typical cross-wave experiments using water, ${ }^{18,20,22,24-26}$ where the wavemaker depth is more than $10 \mathrm{~cm}$, the self-consistency of the NLS models requires forcing frequencies on the order of $10 \mathrm{~Hz}$ or less. ${ }^{23}$ These models cannot be expected to apply to experiments using higher frequencies or larger viscosity fluids and are thus inadequate to describe pattern formation experiments in moderately sized containers, which require higher forcing frequencies (smaller wavelengths). Indeed, several cross-wave experiments in this regime ${ }^{5,7.23}$ have found interesting rotated patterns that deviate from the assumption of (nearly) crosswise orientation made in the NLS models.

An extension of cross-wave theory is needed to cover the parameter range beyond traditional (large) wavemaker experiments with low frequencies and small damping. It is the aim of this paper to take a first logical step toward that end, extending the standard analysis that followed Jones ${ }^{15}$ and, in particular, that of Bernoff $e t a l .{ }^{17}$ by including the effect of surface tension and, most importantly, relaxing the assumption of a narrow forcing region. Other assumptions common to previous cross-wave analyses, ${ }^{15-18,21,27}$ like that of a nearly crosswise orientation, are maintained here. This is done for the sake of comparison, so that the effect of distributed forcing can be clearly seen without the complications of additional new effects. It follows that the model obtained below will not apply to all subharmonic patterns excited by horizontal forcing, in particular those that are very rotated with respect to standard cross-waves. ${ }^{28}$ It does, however, apply to a wide range of low viscosity experiments in the gravity-capillary regime including some results of Porter $a t a l .^{23}$ where typical subharmonic patterns are rotated (i.e., modulated in the downstream direction) but often not excessively so at onset. The model obtained here has the theoretical advantage of allowing direct comparison with the many established results based on NLS models that assume a narrow forcing region.

The main point of this paper is to demonstrate that, in the gravity-capillary parameter regime of interest, distributed forcing is the key to obtaining predictions consistent with experiment. This is confirmed by a series of new experimental results for the onset of crosswaves in containers of various sizes, and by measurements of the spatial and temporal modulations of the onset patterns. In particular, the interesting quasiperiodic solutions predicted by the model in Sec. V, which cycle between one-sided and two-sided excitation, are located experimentally.

\section{BACKGROUND: CROSS-WAVE THEORY}

The most influential approach to the theory of cross-waves was initiated by Jones, ${ }^{15}$ who obtained a cubic NLS equation to describe the slow spatial and temporal evolution of the cross-wave amplitude. Although alternative derivations were presented by other authors that take account of effects like viscosity and mode interactions ${ }^{16-19}$ the underlying assumptions are the same. These are:

1. Damping is small, or absent.

2. Applied forcing is small.

3. Surface tension can be neglected in the free-surface boundary conditions.

4. The downstream variation of the subharmonic waves is slow compared to (a) the crosswise variation (i.e., waves are oriented crosswise, or nearly so) and to (b) the spatial decay of the evanescent harmonic flow field generated by the wavemaker, which provides the forcing mechanism. 
The first two assumptions are critical for obtaining analytical results and are still justified over a range of experimental conditions, like those of Porter et al. ${ }^{23}$ where the NLS models no longer apply. The final two assumptions were motivated by the earlier wavemaker experiments ${ }^{14,18,20,22,24-26}$ that the NLS models were intended to explain, nearly all of which were performed in large tanks of water (more than a meter in length) and at low frequencies (less than $10 \mathrm{~Hz}$ ). These assumptions are not essential to the derivation and must be relaxed if the theory is to be extended to higher frequencies and gravity-capillary waves.

We will focus here on the particular model derived by Bernoff et al. ${ }^{17}$ which includes the leading order effects of viscous damping at the wavemaker, sidewalls, bottom, and free surface. The formulation assumes a planar wavemaker oscillating about $x=0$ according to $x(t)=a \cos (2 \sigma t)$. A fluid with small kinematic viscosity $v$ is confined in a semi-infinite domain with (crosswise) width $W$ and depth $D$. The vertical gravitational acceleration is $g$. The mode number $N$ defines the crosswise wavenumber $k=N \pi / W$. After nondimensionalizing with $k$ and $\sigma$, the system depends on the small forcing parameter $\epsilon=a k \ll 1$, the Reynolds number $\operatorname{Re}=\sigma /\left(\nu k^{2}\right) \gg 1$, and the nondimensional depth $d=k D$. The damped NLS equation obtained in Bernoff et al. ${ }^{17}$ after neglecting the inhomogeneous contribution to the detuning due to the harmonic waves, can be written in terms of the original (fast) space and time variables, as

$$
\begin{aligned}
& i A_{t}=\frac{S}{4} A_{x x}+\left(v-i \gamma_{0}\right) A+\epsilon^{2} \beta|A|^{2} A, \\
& A_{x}=-\epsilon R \bar{A}-\gamma_{1} A \text { at } x=0, \\
& A \rightarrow 0 \text { as } x \rightarrow \infty,
\end{aligned}
$$

where $S=1+2 d / \sinh (2 d), v=\left(\sigma-\sigma_{0}\right) / \sigma_{0}$ with $\sigma_{0}^{2}=g k \tanh (d), \gamma_{0}=2 / \operatorname{Re}+\sqrt{i}[1 / \sinh (2 d)$ $+(2-S) /(N \pi)] / \sqrt{\operatorname{Re}}, \beta=\left(16-9 H^{2}-5 / H^{2}+6 / H^{4}\right) / 16$ with $H=1 / \tanh (d), R=[d(H+3 / H)$ $-2] / S$, and $\gamma_{1}=2 /(S \sqrt{i \operatorname{Re}})$.

Note that, as a result of the separation of lengthscales permitted by assumption 4(b), the waves are forced only via the homogeneous boundary condition (1b). This contrasts with the case of vertically forced Faraday waves (where the forcing is uniformly distributed) and with the theory of Varas and $\mathrm{Vega}^{29}$ where the forcing term appears in the governing equation because it varies on the same scale as the modulation. The appearance of the forcing as a boundary condition has significant consequences for the NSL model, particularly with respect to the dependence of the neutral stability curves on forcing frequency and viscosity. These neutral stability curves are straightforward to obtain from the linearized version of Eqs. (1) by writing $A(x)=A_{0} e^{\kappa x}$. They take the form

$$
g_{\text {rel }}^{c}=\frac{4 \sigma^{2}\left|\kappa+\gamma_{1}\right|}{g k|R|}
$$

with

$$
\kappa^{2}=\frac{4}{S}\left(i \gamma_{0}-v\right), \quad \text { real }(\kappa)<0
$$

where the critical value is expressed in terms of relative acceleration using $g_{\text {rel }}=a(2 \sigma)^{2} / g$. Equation (2) defines a set of neutral stability tongues (indexed by $N$ ) whose minima decrease with increasing forcing frequency $\sigma$ (see Fig. 13 of Porter et al ${ }^{23}$ ). This behaviour is not explained by damping at the container bottom and occurs even if bottom, sidewall, and wavemaker damping are all neglected. It is a result of the separation of lengthscales assumed in the derivation of the NLS models. This assumption is strictly valid only for very small damping (equivalently, very low frequencies) or for very shallow wavemakers. It is doubtful that such a separation of lengthscales holds well enough for a quantitative prediction of the cross-wave threshold, for example, even in some of the experiments to which the NLS models have been applied with some success.

Consider, for instance, the experiments of Underhill et al.$^{22}$ where cross-wave modes $N=5$ and $N=7$ were measured. The critical acceleration did not decrease with forcing frequency (mode number) as predicted, but increased instead. These experiments were performed with a fluid depth of $25.8 \mathrm{~cm}$, which is greater than the wavelengths of the two modes: $12.36 \mathrm{~cm}$ and 
$8.83 \mathrm{~cm}$, respectively. Damping at the bottom is therefore not important. From Figs. 3 and 7 of that reference, critical acceleration values of $0.064 \mathrm{~g}$ for $N=5$ and $0.074 \mathrm{~g}$ for $N=7$ can be extracted, showing a clear increase with increasing forcing frequency-again, something which cannot be explained by the NLS model.

Neutral stability curves were also measured by Shemer and Lichter $^{30}$ for mode numbers 3,5 , 6 , and 7 in a wave tank measuring $120 \mathrm{~cm}$ across. The corresponding wavelengths for these modes are $80 \mathrm{~cm}, 48 \mathrm{~cm}, 40 \mathrm{~cm}$, and $34.3 \mathrm{~cm}$, which should be compared to the depth of $60 \mathrm{~cm}$. From Fig. 2 of that paper, one can obtain estimates for the (minimum) acceleration required to excite these four modes: $0.153 \mathrm{~g}, 0.137 \mathrm{~g}, 0.144 \mathrm{~g}$, and $0.167 \mathrm{~g}$, respectively. In dropping from $N=5$ to $N=3$, there is a $12 \%$ increase in the critical acceleration needed but this can be explained by the fact that the wavelength for $N=3$ exceeds the container depth, leading to an increase in damping at the bottom. In contrast, when mode number is increased between 5 and 7 , where the wavelength is less than the fluid depth, there is a measured increase in critical acceleration, contrary to the NLS model. Although this experiment is a bit harder to interpret than that of Underhill $e t$ al. ${ }^{22}$ because of the segmented wavemaker used, which generates larger dissipation via vortex shedding at the discontinuities, ${ }^{30}$ the increase in critical acceleration with increasing mode number cannot easily be explained by the NLS model, which suggests that the balance between damping and forcing resulting from the assumed separation of lengthscales is not entirely justified.

\section{DERIVATION OF DISTRIBUTED FORCING MODEL}

Here we extend the standard NLS models by deriving an amplitude equation to describe modulated cross-waves that includes the effect of surface tension and does not assume a separation of scales between the downstream modulation of the waves and the extent of the forcing. As in Bernoff $e t a l .,{ }^{17}$ we first consider a semi-infinite domain: $x \geq 0,0 \leq y \leq W,-D \leq z \leq 0$ from a coordinate system oscillating periodically along the $x$ axis with frequency $2 \sigma$ and amplitude $a$. The fluid has density $\rho$, viscosity $\nu$, and surface tension $\Gamma$. The crosswise wavenumber $k_{y}=N \pi / W$ is characterized by the mode number $N$ and the natural trequency $\omega_{0}$ is defined by the inviscid dispersion relation

$$
\omega_{0}^{2}=\left(g k_{y}+\frac{\Gamma}{\rho} k_{y}^{3}\right) \tanh \left(k_{y} D\right) .
$$

The nondimensional forcing, gravity number, capillary number, and Reynolds number are defined by

$$
\epsilon=a k_{y}, \quad G_{0}=\frac{g k_{y}}{\omega_{0}^{2}} \quad \Gamma_{0}=\frac{\Gamma k_{y}^{3}}{\rho \omega_{0}^{2}}, \quad \operatorname{Re}=\frac{\omega_{0}}{\nu k_{y}^{2}},
$$

respectively. Note that $G_{0}+\Gamma_{0}=1 / \tanh (d)=H$.

The system will be forced near resonance, so we write $\omega \equiv \sigma / \omega_{0}=1+v$ with v small. After rescaling length and time with $k_{y}$ and $\omega_{0}$, respectively, we have the following equations in the oscillating reference frame:

$$
\begin{aligned}
\mathbf{v}_{t}+\mathbf{v} \cdot \nabla \mathbf{v} & =-\nabla p+\frac{1}{\operatorname{Re}} \Delta \mathbf{v}+4 \omega^{2} \epsilon \cos (2 \omega t) \mathbf{e}_{x}, \\
\nabla \cdot \mathbf{v} & =0 .
\end{aligned}
$$

where $p-G_{i z}$ is the pressure, $\nabla=\mathbf{e}_{x} \partial_{x}+\mathbf{e}_{y} \partial_{y}+\mathbf{e}_{z} \partial_{z}, \Delta=\partial_{x}^{2}+\partial_{y}^{2}+\partial_{z}^{2}$, and $\mathbf{e}_{x}, \mathbf{e}_{y}$, and $\mathbf{e}_{z}$ are unit vectors aligned along the coordinate axes. No-slip boundary conditions are imposed on the solid walls. The free surface $z=f(x, y)$ satisfies the following stress balance and kinematic conditions: ${ }^{31}$

$$
\begin{aligned}
p-G_{0}\left(\hat{\mathbf{n}} \cdot \mathbf{e}_{z}\right) f+2 \Gamma_{0} M & =\frac{1}{\operatorname{Re}} \hat{\mathbf{n}} \cdot\left[\nabla \mathbf{v}+\nabla \mathbf{v}^{T}\right] \cdot \hat{\mathbf{n}}, \\
\hat{\mathbf{n}} \times\left[\nabla \mathbf{v}+\nabla \mathbf{v}^{T}\right] \cdot \hat{\mathbf{n}} & =0 \\
\left(\mathbf{v}-f_{i} \mathbf{e}_{z}\right) \cdot \hat{\mathbf{n}} & =0
\end{aligned}
$$


where $M=\bar{\nabla} \cdot \hat{\mathbf{n}} / 2$ is the mean curvature of the surface, $\bar{\nabla}=\mathbf{e}_{x} \partial_{x}+\mathbf{e}_{y} \partial_{y}$ denotes the horizontal gradient operator, and $\hat{\mathbf{n}}$ is given by

$$
\hat{\mathbf{n}}=\frac{(-\bar{\nabla} f, 1)}{\sqrt{1+|\bar{\nabla} f|^{2}}} .
$$

In the quasipotential approximation, the bulk of the fluid is taken to be irrotational and inviscid with $u=\nabla \phi$. The velocity potential $\phi$ then satisfies the Laplace equation

$$
\Delta \phi=0 .
$$

Boundary layers at the walls and free surface are assumed to be linear. Standard analysis for the boundary layer at the walls provides the velocity, and matching the normal component across the layer leads to the condition. ${ }^{17}$

$$
\left(\hat{\phi}_{\Omega}\right)_{n}=-\frac{1}{\sqrt{i \Omega \mathrm{Re}}}\left(\hat{\phi}_{\Omega}\right)_{n n}
$$

where the subscript $n$ indicates a derivative in the normal direction and $\hat{\phi}_{\Omega}(\mathbf{x})$ denotes the temporal Fourier component of $\phi(\mathbf{x}, t)$ oscillating as $\exp \{i \Omega t\}$; hereafter, the subscript $\Omega$ will be suppressed to simplify notation. The boundary layer at the free surface provides linear viscous corrections to both the kinematic compatibility condition and normal stress at the interface. We apply the following boundary conditions, ${ }^{8}$ valid to order $O\left(\epsilon^{2} \mathrm{Re}^{-1 / 2}+\epsilon^{3}\right)$

$$
\begin{aligned}
& f_{t}-\phi_{z}=\frac{2}{\operatorname{Re}} \underline{\Delta} f-\bar{\nabla} \phi \cdot \bar{\nabla} f, \\
& \phi_{t}+\frac{G_{0} f}{\sqrt{1+|\bar{\nabla} f|^{2}}}-\Gamma_{0} \bar{\nabla} \cdot\left[\frac{\bar{\nabla} f}{\sqrt{1+|\bar{\nabla} f|^{2}}}\right]=4 \epsilon \cos (2 \omega t) x+\frac{2}{\operatorname{Re}} \underline{\Delta} \phi-\frac{1}{2}|\nabla \phi|^{2} .
\end{aligned}
$$

Expanding about $z=0$ and retaining terms up to cubic order yields the following system

$$
\Delta \phi=0
$$

with boundary conditions at the surface $z=0$,

$$
\begin{aligned}
& f_{i}-\phi_{z}=\frac{2}{\operatorname{Re}} \underline{\Delta f}+f \phi_{z \bar{z}}-\bar{\nabla} \phi \cdot \bar{\nabla} f+\frac{1}{2} \phi_{z z z} f^{2}-f \bar{\nabla} \phi_{\bar{z}} \cdot \bar{\nabla} f, \\
& \phi_{t}+G_{0} f-\Gamma_{0} \underline{\Delta} f=4 \epsilon \cos (2 \omega t) x-\frac{2}{\operatorname{Re}} \phi_{z z}-\frac{1}{2}|\nabla \phi|^{2}-f \phi_{z t}-\frac{1}{2} \phi_{z z t} f^{2} \\
& -f \nabla \phi_{z} \cdot \nabla \phi+\frac{1}{2} G_{i} f|\bar{\nabla} f|^{2}-\frac{1}{2} \Gamma_{i} \bar{\nabla} \cdot\left[\bar{\nabla} f|\bar{\nabla} f|^{2}\right],
\end{aligned}
$$

where $\underline{\Delta}=\partial_{x}^{2}+\partial_{y}^{2}$, and at the boundaries

$$
\begin{array}{ll}
\hat{\phi}_{x}=\frac{-1}{\sqrt{i \Omega \mathrm{Re}}} \hat{\phi}_{x x} & \text { at } \quad x=0, \\
\phi \rightarrow 0 & \text { as } \quad x \rightarrow \infty . \\
\hat{\phi}_{y}=\frac{\mp 1}{\sqrt{i \Omega \mathrm{Re}}} \hat{\phi}_{y y} \quad \text { at } \quad y=0, N \pi, \\
\hat{\phi}_{z}=\frac{-1}{\sqrt{i \Omega \mathrm{Re}}} \hat{\phi}_{z z} \quad \text { at } \quad z=-d .
\end{array}
$$




\section{A. Scaling}

We expand ${ }^{15,17}$ dependent variables in powers of $\epsilon$

$$
\begin{aligned}
& \phi=\epsilon \phi^{(1)}+\epsilon^{2} \phi^{(2)}+\epsilon^{3} \phi^{(3)}+\ldots . \\
& f=\epsilon f^{(1)}+\epsilon^{2} f^{(2)}+\epsilon^{3} f^{(3)}+\ldots, \\
& v=\epsilon^{2} v^{(2)}
\end{aligned}
$$

and introduce slow spatial and temporal scales

$$
(X, Y)=\epsilon(x, y), \quad T_{1}=\epsilon t, \quad T_{2}=\epsilon^{2} t .
$$

We further assume ${ }^{17}$ that $\epsilon \operatorname{Re}^{1 / 2} \equiv \delta^{-1}$ is $O(1)$ and solve the system (12) at successive orders in $\epsilon$. Details of this procedure, which leads to a solvability condition that gives the amplitude equations describing subharmonic waves, can be found in the Appendix.

\section{B. Leading order solution and harmonic flow}

At leading order in $\epsilon$ one obtains the inhomogeneous linear problem from Eqs. (12):

$$
\Delta \phi^{(1)}=0,
$$

with boundary conditions at the surface $z=0$ given by

$$
\begin{aligned}
& f_{t}^{(1)}-\phi_{\bar{c}}^{(1)}=0, \\
& \phi_{t}^{(1)}+G_{0} f^{(1)}-\Gamma_{0} \underline{\Delta} f^{(1)}=4 \cos (2 \omega t) x,
\end{aligned}
$$

and at the boundaries

$$
\begin{aligned}
& \phi_{x}^{(1)}=0 \quad \text { at } \quad x=0, \\
& \phi^{(1)} \rightarrow 0 \quad \text { as } \quad x \rightarrow \infty, \\
& \phi_{y}^{(1)}=0 \quad \text { at } \quad y=0, N \pi, \\
& \phi_{z}^{(1)}=0 \quad \text { at } \quad z=-d .
\end{aligned}
$$

We distinguish three distinct contributions at this order by writing

$$
\phi^{(1)}=\left[-i x+\phi^{H}\right] e^{2 i \omega t}+\phi^{S W} e^{i(\omega t}+c . c .,
$$

where $\omega=1+\epsilon^{2} v^{(2)}$. The second (harmonic) term can be expressed using cosine transform methods as

$$
\phi^{H}=-\frac{4}{\pi} \int_{C} \frac{e^{-q x} \cos [q(z+d)]}{q^{2}\left[4 \cos (q d)+\left(G_{0} q-\Gamma_{0} q^{3}\right) \sin (q d)\right]} d q .
$$

where $C$ is a path from $-i \infty$ to $i \infty$ returning clockwise along a semicircle of infinite radius in the right half-plane. This expression, which for simplicity neglects corrections coming from the detuning $v$, contains two contributions (see Havelock $\mathrm{k}^{32}$ ). The first one is a harmonic traveling wave of the form $\phi^{H W}=B\left(X, T_{1}, T_{2}\right) \exp \{i k x\} \cosh [k(z+d)]$ corresponding to the complex pole $q=i k$ with $k$ given here by the dispersion relation:

$$
\left(G_{0} k+\Gamma_{i} k^{3}\right) \tanh (k d)=4 .
$$


In the treatment that follows we will neglect the role of the hamonic waves. The second contribution in Eq. (19) is an evanescent field referred to as the oscillating bulk flow. ${ }^{29}$ It can be approximated, except in a narrow region near the walls, by

$$
\phi^{B F}(x, z)=-\frac{2 i}{d} \sum_{n=0}^{\infty} \frac{(-1)^{n}}{q_{n}^{2}} e^{-q_{n} x} \cos \left[q_{n}(z+d)\right],
$$

where $q_{n} d=\pi(n+1 / 2)$. This simplified expression will be used in numerical computations. Note that the oscillating bulk flow $\phi^{B F}$, which exhibits multi-exponential decay in $x$, extends over a lengthscale $x \sim d$.

\section{Solvability condition for cross-waves}

The general form of the solvability condition is given in Eq. (A7). To apply this, we separate vertical and horizontal dependence by writing

$$
\begin{aligned}
& \phi^{\zeta W}=A(X, T) \frac{\cosh (z+d)}{\cosh (d)} \Psi(x, y), \\
& \tilde{\phi}^{\dagger}=\frac{\cosh (z+d)}{\cosh (d)} \bar{\Psi}(x, y) .
\end{aligned}
$$

where $\phi^{\dagger}=\phi^{\dagger} \exp \{-i t\}$ and $f^{\dagger}$, which also oscillates as $\exp \{-i t\}$, are (resonant subharmonic) solutions of the homogeneous linear problem (17) with no forcing. Then, to leading order, at $z=0, f^{\dagger}=i H^{-1} \phi^{\dagger}$ and $f^{s W}=-i H^{-1} \phi^{S W}$, where $f^{S W} \exp \{i t\}$ is the corresponding subharmonic component of the free surface motion. By taking into account

$$
\int_{-d}^{0} \frac{\cosh ^{2}(z+d)}{\cosh ^{2}(d)} d z=\frac{1}{2 H}\left(\frac{2 d}{\sinh (2 d)}+1\right) .
$$

we integrate over the vertical coordinate to obtain the solvability conditions at second order:

$$
\begin{aligned}
& +\frac{i}{H} \int_{z=0} \tilde{\phi}^{\dagger}\left[2 \phi_{T_{1}}^{S W}+i S\left(\phi_{x X}^{S W}+\phi_{y Y}^{S W}\right)+\frac{i}{\epsilon} \phi^{S W} \sum_{\text {edges }} e^{-\sqrt{i \operatorname{Re} \xi_{1}}}\right] d x d y \\
& +\frac{i}{2 H} \int_{z=0} \tilde{\phi}^{\dagger}\left\{\left[2 \phi_{z z}^{B F}-\left(H+3 H^{-1}\right) \phi_{z}^{B F}\right] \widehat{\phi}^{S W}+H \bar{\nabla} \phi_{z}^{B F} \cdot \bar{\nabla} \bar{\phi}^{S W}\right\} d x d y \\
& +\frac{S}{2 H} \int_{x=\bar{z}=0} \tilde{\phi}^{\dagger}\left(-\phi_{X}^{S W}-\frac{\delta}{\sqrt{i}} \phi_{x x}^{S W}\right) d y+\frac{1}{2} \int_{z=-d} \tilde{\phi}^{\dagger}\left(-\frac{\delta}{\sqrt{i}} \phi^{S W}\right) d x d y-\frac{S}{2 H} \int_{y=N \pi, z=0} \tilde{\phi}^{\dagger}\left(-\phi_{Y}^{S W}+\frac{\delta}{\sqrt{i}} \phi_{y \psi}^{S W}\right) d x \\
& +\frac{S}{2 H} \int_{y=\bar{c}=0} \tilde{\phi}^{\dagger}\left(-\phi_{Y}^{S W}-\frac{\delta}{\sqrt{i}} \phi_{y y}^{S W}\right) d x=0
\end{aligned}
$$

and at third order:

$$
\frac{1}{H} \int_{z=0} \tilde{\phi}^{\dagger}\left[2 \phi_{T_{2}}^{S W}+2 i v^{(2)} \phi^{S W}+4 \delta \phi^{S W}+i \frac{S}{2} \phi_{X X}^{S W}\right] d x d y=0
$$

with

$$
S=1+\frac{2 d}{\sinh (2 d)}+2 \Gamma_{0} \tanh (d)
$$

Equation (19) must be used to evaluate the terms at $x=z=0$ to ensure that the terms involving $\phi_{x z}$ and $\phi_{x z}$, vanish (being given by integrals over an odd function of $q$ with no pole at the origin).

To this point, no assumption has been made about the orientation of the subharmonic waves. We now focus on cross-waves of the form $\Psi(x, y)=\cos (y)$, permitting only slow modulation in the 
$x$ direction. After integrating Eqs. (24) and (25) over $y$ and combining the results, we find

$$
\begin{aligned}
0 & =2 i \int_{0}^{\approx}\left[\epsilon A_{T_{1}}+\epsilon^{2} A_{T_{2}}+\left(i v+\gamma_{0}\right) A+i \epsilon^{2} \frac{S}{4} A_{X X}\right] d x \\
& +\frac{i}{2} \epsilon \bar{A} \int_{z=0}\left[2 \phi_{z \bar{z}}^{B F}-\left(H+3 H^{-1}\right) \phi_{z}^{B F}\right] d x+\left.\epsilon \frac{S}{2} A_{X}\right|_{x=0}+\left.\frac{1}{\sqrt{i \mathrm{Re}}} A\right|_{x=0},
\end{aligned}
$$

where, as in Eqs. (1),

$$
\gamma_{0}=\frac{2}{\operatorname{Re}}+\sqrt{\frac{i}{\operatorname{Re}}}\left(\frac{1}{\sinh (2 d)}+\frac{2-S}{N \pi}\right) .
$$

The terms in Eq. (27) must be considered in light of the definition of $X=\epsilon x$. The first integral is formally divergent unless the integrand, which is independent of $x$, vanishes for all $X$. This condition provides the main differential equation to be satisfied. The final two terms come from a boundary term evaluated at $x=0$ and a rapidly decaying free surface velocity contribution (see Eq. (A3)), and thus should be interpreted as a boundary condition at $X=0$. The second term can contribute either to the differential equation or to the boundary condition depending on the assumptions made about the bulk flow $\phi^{B F}$. If $\phi^{B F}$ is assumed to vary rapidly, on the fast lengthscale $x$, then the second term can also be integrated over $x$. The result, which is proportional to $\bar{A}$, is added to the boundary condition at $X=0$ since the principal contribution to the integral comes from $x \sim O(1)$, or $X \ll 1$. In this case, after reconstituting time derivatives with $A_{t}=\epsilon A_{T_{1}}+\epsilon^{2} A_{T_{2}}$, returning to the fast lengthscale using $X=\epsilon x$, and including the cubic nonlinearity (see the Appendix), we have the following system to determine the slowly varying amplitude $A(x, t)$ :

$$
\begin{aligned}
& i A_{t}=\frac{S}{4} A_{x x}+\left(v-i \gamma_{0}\right) A+\epsilon^{2} \beta|A|^{2} A, \\
& A_{x}=-\epsilon R \bar{A}-\gamma_{1} A \text { at } x=0, \\
& A \rightarrow 0 \text { as } x \rightarrow \infty,
\end{aligned}
$$

with

$$
R=\frac{i}{S} \int_{z=0}\left[2 \phi_{z z}^{B F}-\left(H+3 H^{-1}\right) \phi_{z}^{B F}\right] d x=\frac{1}{S}\left[d\left(H+3 H^{-1}\right)-2\right]
$$

and

$$
\gamma_{1}=2 /(S \sqrt{i \operatorname{Re}}) \text {. }
$$

This is simply the extension of the amplitude equations (1) of Bernoff $e f$ al ${ }^{17}$ to the gravity-capillary regime, reflected in the definition of $S$ in Eq. (26). It applies only if $g(x)$ decays much faster with distance from the endwall than $A(x, t)$, as will be the case, for example, if the fluid (wavemaker in other experimental configurations) is shallow.

On the other hand, if the fluid depth $d$ is larger than or comparable to the extent of the subharmonic field $A(x, t)$ then the bulk flow $\phi^{B F}$ will extend into the interior and cannot be integrated out. ${ }^{29}$ In this more general case, when $\phi^{B F}$ is taken to vary on the same slow lengthscale as the amplitude $A$, the second integral should be combined with the first. The combined integrand, which is independent of $x$, is then set to zero to give the following system with distributed forcing:

$$
\begin{aligned}
& i A_{t}=\frac{S}{4} A_{x x}+\left(v-i \gamma_{0}\right) A+\epsilon g(x) \bar{A}+\epsilon^{2} \beta|A|^{2} A, \\
& A_{x}=-\gamma_{1} A \text { at } x=0, \\
& A \rightarrow 0 \quad \text { as } \quad x \rightarrow \infty,
\end{aligned}
$$


where

$$
g(x)=-\left.\frac{i}{4}\left[2 \phi_{z z}^{B F}-\left(H+3 H^{-1}\right) \phi_{\bar{z}}^{B F}\right]\right|_{z=0} .
$$

Note that $\gamma_{1}$, which accounts for damping at the endwall, is due solely to the contribution (A3).

\section{ONSET OF CROSS-WAVES}

If the effect of the harmonic surface waves, $\phi^{H W}$, is neglected, then cross-waves are driven entirely by the oscillating bulk flow $\phi^{B F}$, which itself is generated by the motion of the boundary (wavemaker) at $x=0$. Whether $\phi^{B F}$ is assumed to decay rapidly or not in the downstream direction is a critical step in the derivation of the amplitude equations that determines the balance between forcing and damping required for the excitation of subharmonic waves. If $\phi^{B F}$ decays rapidly then cross-waves are forced via a homogeneous boundary condition as in Eqs. (29). If $\phi^{B F}$ extends into the interior of the domain then a distributed parametric forcing term $g(x) \bar{A}(x, t)$ appears as in Eqs. (32). We examine here the differences between these two systems.

Neutral stability curves can be obtained directly from Eqs. (29) by looking for steady solutions of the form $A=A_{0} \exp \{\kappa x\}$. These curves are defined by Eq. (2) with $S$ given by Eq. (26). The distributed forcing model (32) must be solved numerically. The neutral stability curves predicted by the two systems are shown together in Fig. 1 for parameters appropriate to the experiments of Porter $e t$ al. ${ }^{23}$ Note that Eqs. (29) give a much lower threshold for the excitation of cross-waves than Eqs. (32). With a forcing frequency of $50 \mathrm{~Hz}$, for example, Eqs. (29) predict that the $N=27$ mode will onset first at a critical acceleration of $g_{\text {rel }} \simeq 0.0447$ while Eqs. (32) indicate that the $N=26$ mode emerges first at a critical acceleration of $g_{\text {rel }} \simeq 0.348$.

The two systems are expected to agree only when a separation of lengthscales between the decay of $g(x)$ and the modulation of $A(x, t)$ is justified. This requires either a shallow depth $d$ (recall that $d$ characterizes the extent of $g(x)$ ), or small viscosity or frequency, where the modulation lengthscale (characterized by $\kappa$ of Eq. (2)) will generally be larger (depending on detuning) than $d$. When a separation of lengthscales between $g(x)$ and $A(x, t)$ is not justified, the behaviour of the two systems differs markedly. There is a different balance between forcing and damping and, consequently, a different scaling with respect to forcing frequency and viscosity, as shown in Figs. 1 and 2, respectively.

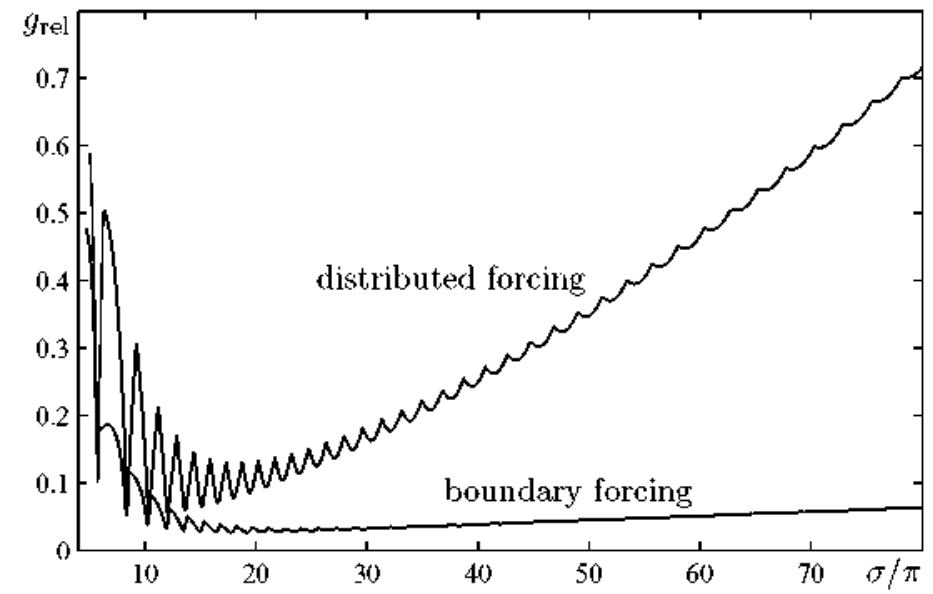

FIG. 1. Comparison of onset values for forcing frequencies up to $80 \mathrm{~Hz}$ predicted by the model (29) with boundary forcing and the model (32) with distributed forcing. Remaining parameters are $v=5 \mathrm{cSt}, \Gamma=19.7 \mathrm{dyne} / \mathrm{cm}, \rho=0.913 \mathrm{~g} / \mathrm{cm}^{3}$, $g=980 \mathrm{~cm} / \mathrm{s}^{2}, D=5 \mathrm{~cm}$, and $W=9 \mathrm{~cm}$. 


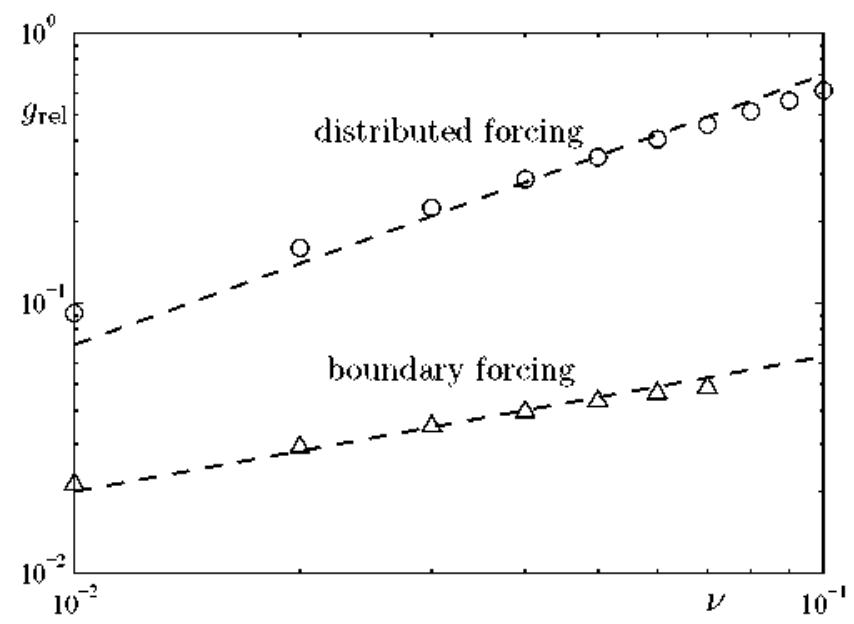

FIG. 2. Scaling of minimun onset value versus viscosity (in cSt) for mode $N=26$ (which is resonantly forced near $50 \mathrm{~Hz}$ ) using Eqs. (29) and (32). Remaining parameters are as in Fig. 1. The dashed curves show the scalings $\xi_{\text {rel }} \sim v$ (distributed forcing case) and 3 rel $\sim 1^{1 / 2}$ (boundary forcing case).

\section{A. Secondary cross-wave modes}

The consequences of assuming a separation of scales that places the entire effect of the forcing at the boundary are qualitative as well as quantitative. In the boundary forced system (29), the neutral stability curve, with fixed parameters and a given mode number $N$, is uniquely defined by Eq. (2). This is not the case in system (32), which allows for multiple solutions at the same parameters and mode number. An example of this multiplicity is shown in Fig. 3, which shows the first three neutral stability curves for the $N=26$ mode $\left(\omega_{0} / \pi \approx 50 \mathrm{~Hz}\right)$ and the corresponding eigentunction profiles.

To understand the multiplicity illustrated in Fig. 3, it is useful to split the domain into a supercritical "standing wave" region, where waves are locally supported by the forcing, and a subcritical region, where they are not. We define the turning point $x_{\mathrm{tp}}$ as the place where $\epsilon g\left(x_{\mathrm{tp}}\right)$ is just enough, supposing it were uniform in space, to excite subharmonic (Faraday) waves. Ignoring spatial modulations, this condition becomes $\epsilon g\left(x_{\mathrm{tp}}\right)=\left|v-i \gamma_{0}\right|$. Turning points defined in this way are marked with an asterisk in the right panel of Fig. 3, which shows the utility of this point in separating the oscillatory part of the solution from the exponentially decaying part. This separation
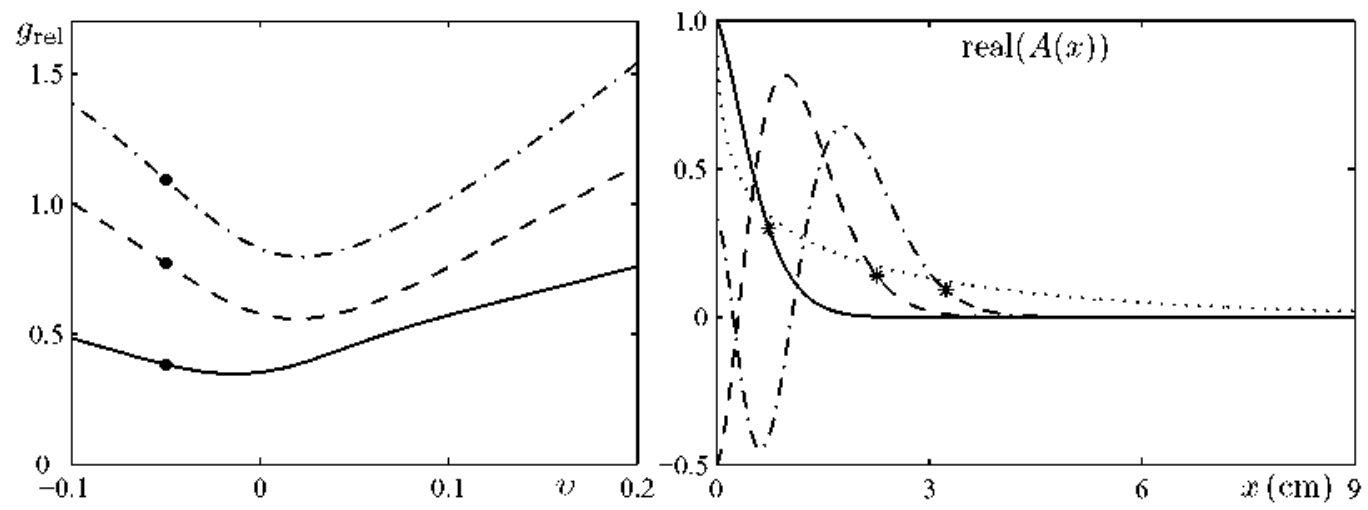

FIG. 3. Multiple solutions exist in Eqs. (32) with the same crosswise mode numbet. Left: First three neutral stability curves for $N=26$. Right: $\operatorname{Real}(A(x)$ ) at the three marked points cortesponding to $v=-0.05$ and 3 rel $=0.3844$ (first mode, solid curve), 0.7726 (second mode, dashed curve), and 1.093 (third mode, dash-dotted curve). An asterisk marks the turning point for each mode (discussed in the text), while the doted curve shows the protile of the forcing function $g(x)$ detined by Eq. (33). Remaining paraneters are as in Fig. 1. 


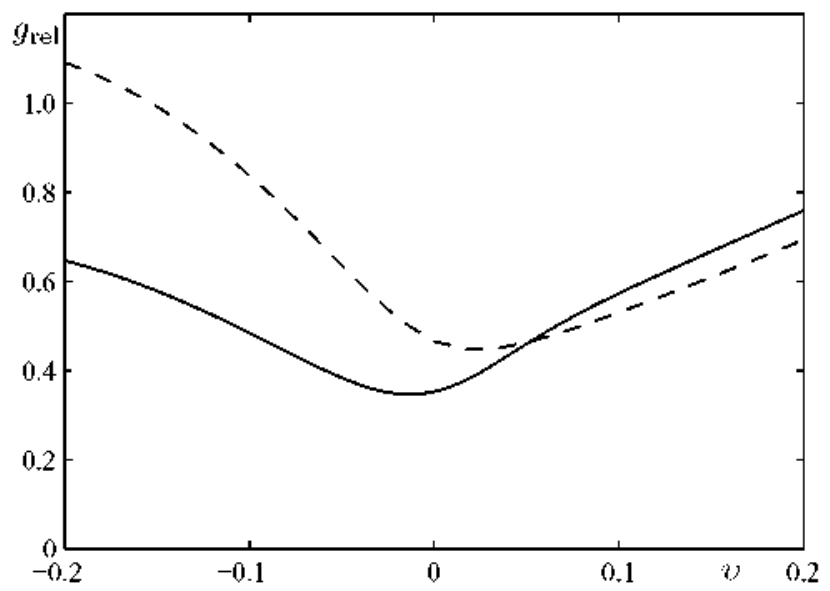

FIG. 4. Comparison of neutral stability curves for $N=26$ with Eqs. (32) (solid curve) and with sinjple Dirichlet boundary conditions instead of Eq. (32b) (dashed curve). Remaining parameters are as in Fig. 1.

is clearest if the solution is relatively localized, as it is with negative detuning. Solutions with positive detuning tend to oscillate more rapidly in $x$ and to extend over a larger region. Whatever the detuning, there is a family of secondary modes that may be described as having successively more oscillations in the supercritical region.

The existence of secondary cross-wave modes with the same mode number $N$ depends on the finite width of the forced (supercritical) region, which scales as $d$. Loosely speaking, the different behaviour of $A(x, t)$ in the supercritical region, where energy input maintains something close to a standing wave, and the underforced subcritical region, where $A(x, t)$ resembles a damped traveling wave, leads to a type of matching condition to be satisfied at the boundary. This can be seen most clearly in a simplified model such as the one proposed by Porter $e t$ al $^{33}$

Modes with more oscillations in the supercritical region require greater forcing than the primary mode and are thus expected only to affect dynamics above onset. The more extended the forcing $g(x)$ is, the sooner these secondary modes appear with increasing acceleration and the more likely they are to play a role in selection of the initial cross-wave pattern. This type of complexity cannot be captured by the boundary condition forcing of Eqs. (29) that ignores the finite width of $g(x)$.

\section{B. Dirichlet boundary conditions}

Unlike boundary forced NLS models such as Eqs. (29), the distributed forcing mechanism in Eqs. (32) permits a variety of alternative boundary conditions to be sensibly applied, including simple Dirichlet or Neumann conditions. In particular, the application of Dirichlet boundary conditions, $A(x, t)=0$ at $x=0$, should be relevant to experiments with fixed contact lines. Thresholds obtained with Dirichlet boundary conditions are not very different, in fact, from those obtained with Eq. (32b). As seen in Fig. 4, an important qualitative difference is the value of detuning where the minimum occurs. In contrast to Eq. (32b), Dirichlet boundary conditions typically lead to a positive detuning value at onset and, thus, an eigenfunction that is modulated more rapidly in $x$ than those found in Sec. IV. This more rapid modulation is in better agreement with the experimentally observed patterns and, as will be seen in Sec. VI, additional evidence suggests that Dirichlet boundary conditions are often closer to the experimental ones.

\section{FINITE-SIZE EFFECTS}

In a laboratory experiment, the fluid must, of course, be confined to a finite domain. When the length $L$ of the horizontally vibrated container considered here is large compared to the extent of the forcing, which is on the order of the depth $d$, and to the extent of the cross-wave field $A(x, t)$, 
which depends also on damping and detuning, then the excitation of subharmonic cross-waves can be treated separately at each side of the container and the analysis of Sec. III applies. If this is not the case, because $d$ cannot be considered small compared to $L$, or because the cross-waves generated at opposing ends extend far enough into the interior to interact with each other, then the full domain must be considered from the beginning.

The solution for the bulk flow when $L$ is not large compared to $d$ is given by

$$
\phi^{B F}=\frac{2 i}{d} \sum_{n=0}^{\infty} \frac{(-1)^{n} \sinh \left(q_{n} x\right)}{q_{n}^{2} \cosh \left(q_{n} L / 2\right)} \cos \left[q_{n}(z+d)\right],
$$

which is antisymmetric under reflection $x \rightarrow-x$. No-slip boundary conditions are applied at $x= \pm L / 2$ and Eqs. (32) are replaced by

$$
\begin{aligned}
& i A_{t}=\left(v-i \gamma_{0}\right) A+\frac{S}{4} A_{x x}+\epsilon g(x) \bar{A}+\epsilon^{2} \beta|A|^{2} A, \\
& A_{x} \pm \gamma_{1} A=0 \quad \text { at } \quad x= \pm L / 2,
\end{aligned}
$$

where $g(x)$ is again given by $\mathrm{Eq}$. (33).

In contrast to the case of a semi-infinite domain, where steady solutions $A(x)$ can be sought (corresponding to $2 \pi / \sigma$-periodic subharmonic waves in the original problem), the interaction of opposing wavetrains in the two-sided problem leads, in general, to modulated (quasiperiodic) solutions. ${ }^{28,33}$ We search for Hopf bifurcations with the ansatz, ${ }^{29}$

$$
A(x, t)=A^{+}(x) e^{i \varpi t}+A^{-}(x) e^{-i \omega t},
$$

which leads to the following system:

$$
\begin{aligned}
& \frac{S}{4} A_{x x}^{ \pm}+\left[v \pm\left[-i \gamma_{0}\right] A^{ \pm}+\epsilon g(x) \bar{A}^{\mp}=0,\right. \\
& A_{x}^{ \pm}-\gamma_{1} A^{ \pm}=0 \quad \text { at } \quad x=-L / 2, \\
& A_{x}^{ \pm}+\gamma_{1} A^{ \pm}=0 \quad \text { at } \quad x=L / 2,
\end{aligned}
$$

which is equivariant under the symmetry $A^{ \pm} \rightarrow A^{\mp}, m \rightarrow-\infty$. Figure 5 shows the neutral stability curve and associated Hopf frequency for the mode $N=16$ in a square container of length $L=9 \mathrm{~cm}$. Note that the effect of the interaction is larger for positive detuning. Here, when (half) the forcing frequency $\sigma$ is greater than the resonant frequency $\omega_{0}$, the pattern compensates with greater modulation in $x$ and penetrates further into the interior, which increases the effect of interaction and, thus, the Hopf frequency. For negative detuning, the tendency is for the patterns to remain very localized near the endwalls with fewer oscillations in $x$. The interaction between the two opposing sides is therefore weaker and the Hopf frequency is smaller.

A modulated solution corresponding to Fig. 5 with $v=0.1$ is illustrated in Fig. 6 . Note that this quasiperiodic solution switches, on a slow timescale, between one-sided excitation concentrated on the left and one-sided excitation concentrated on the right. Periods of balanced excitation exist between these.

\section{COMPARISON WITH EXPERIMENTAL RESULTS}

In this section, we present experimental measurements of the threshold for subharmonic instability, and of the spatial and (when present) temporal modulations of the corresponding patterns near onset. These are compared with the predictions of Secs. IV and V.

\section{A. Experimental set-up}

The experimental apparatus used to obtain the results reported here is sketched in Fig. 7. In this diagram, $\mathrm{C}$ denotes the experimental container, which is an open transparent square acrylic box 


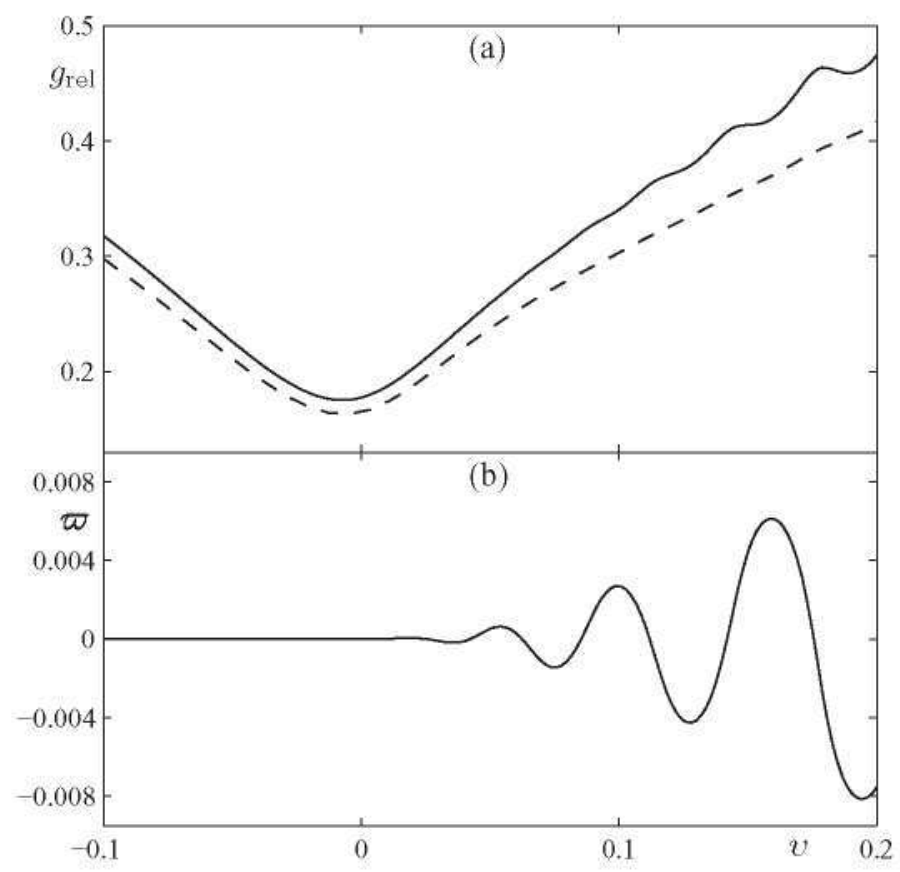

FIG. 5. (a) Neutral stability curve and (b) Hopf frequency $w$ determined by Eqs. (37) for $L=9 \mathrm{~cm}$ and $N=16$ as a function of detuning $v$. For comparison, the dotted curve in (a) shows the neutral stability stability curve for Eqs. (32) corresponding to $L=\infty$. Due to the symmetry of Eqs. (37) there is an associated solution branch (not shown in (b)) with $\varpi \rightarrow-\varpi$. Remaining parameters are as in Fig. 1 .

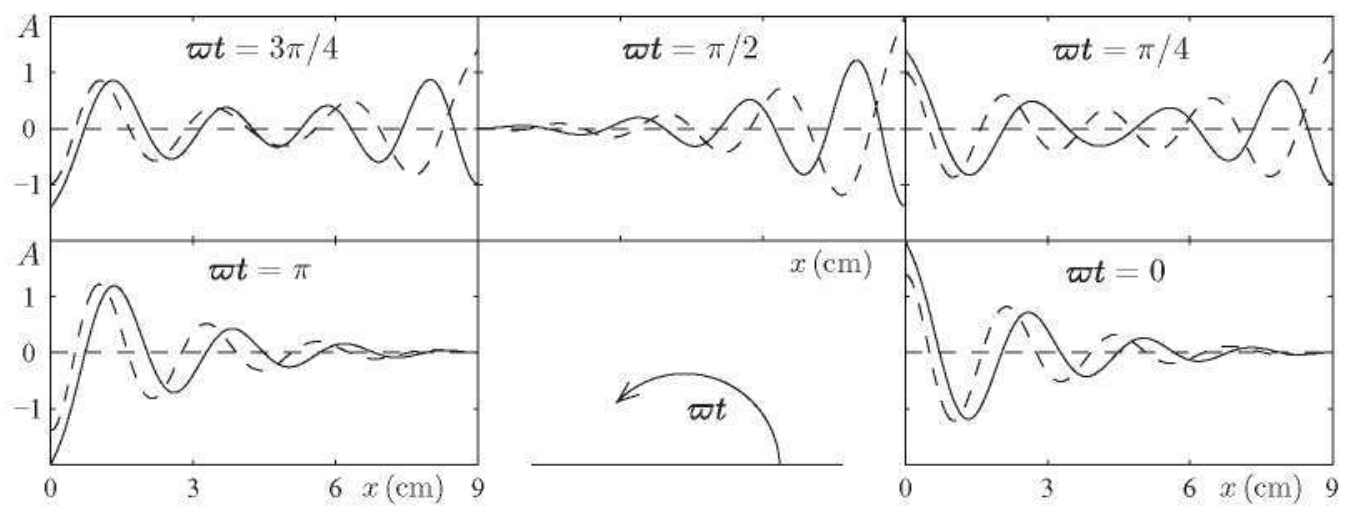

FIG. 6. Modulated solution of Eqs. (37) corresponding to the neutral stability curve in Fig. 5 for $L=9 \mathrm{~cm}$ with $v=0.1$. Solid lines denote real $(A(x))$, with $A$ defined by Eq. (36), while dashed lines denote imag $(A(x))$. The modulation period is about $24.3 \mathrm{~s}$.

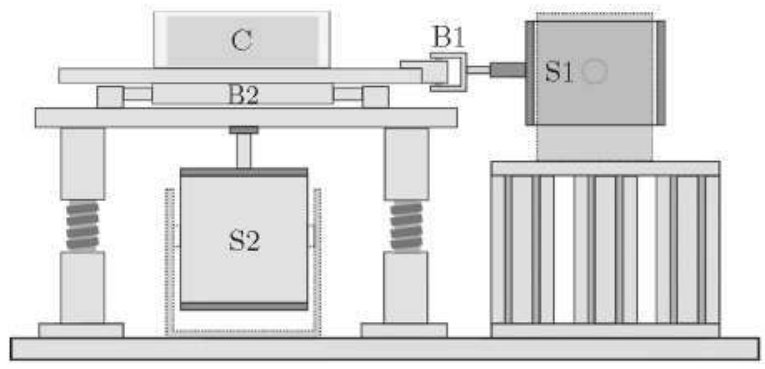

FIG. 7. Diagram of the experimental set-up. The container of fluid (C) is driven horizontally by the electromagnetic shaker (S1). The vertically oriented shaker (S2) is not used here. 
designed to hold a $5 \mathrm{~cm}$ deep layer of fluid with an interior cross-section of $9 \mathrm{~cm}, 15 \mathrm{~cm}$, or $20 \mathrm{~cm}$. A sharp edge (step) at the top of the container is used to pin the contact line when desired, and is coated with an anti-wetting agent (3M Novec EGC-1700). One minor difference between the $9 \mathrm{~cm}$ container and the other two is that in this smaller container the step extends around all four sides, while the others have a step only on the endwalls (lateral walls are flat). The container is attached to a horizontal platform that is driven by an electromagnetic shaker \$1 (VTS-100). The platform and container slide together in rigid fashion along a dual linear sliding bearing system B2. The diagram also shows a second electromagnetic shaker S2 (VTS-150) which is used to vibrate a second, heavier platform beneath $B 2$. When activated, this vertically oriented shaker $S 2$ can be used in combination with $S 1$ to study combined vertical and horizontal excitation. The bearing joint B1 is designed to reduce, as much as possible, the coupling between these two modes of excitation. Results of an investigation of combined excitation will be reported elsewhere. In this paper, we are interested only in cross-waves so only the horizontal shaker $\mathrm{S} 1$ is used.

The working fluid is DC200 silicone oil with kinematic viscosity of $5 \mathrm{cSt}$ or $10 \mathrm{cSt}$. At $25^{\circ} \mathrm{C}$, these two fluids have densities of $0.913 \mathrm{~g} / \mathrm{cm}^{3}$ and $0.933 \mathrm{~g} / \mathrm{cm}^{3}$, respectively, and characteristic surface tension values of $19.7 \mathrm{dyne} / \mathrm{cm}$ and $20.1 \mathrm{dyne} / \mathrm{cm}$. The acceleration experienced by the fluid is measured with an accelerometer (Freescale MMA7361L) mounted on the wall of the container.

Measurements of the surface wave pattern are obtained using a free surface synthetic Schlieren technique described by Moisy et al. ${ }^{34}$ based on analyzing the refracted image of a random pattern of dots placed at the bottom of the container. A high speed camera (Redlake MotionPro X3) takes 15 snapshots per forcing period (30 images for each period of the subharmonic response); the spatial resolution is $0.129 \mathrm{~mm}$ for the $9 \mathrm{~cm}$ container and $0.198 \mathrm{~mm}$ for the $20 \mathrm{~cm}$ container.

The experiments reported here are similar to those described in more detail in Porter $e$ tal. ${ }^{23}$ and similar experimental challenges affect the accuracy of the results. Most notable is the difficulty in maintaining uniform boundary conditions over long periods, or from one experiment to the next. The fluid motion is most vigorous at the endwalls and, especially in the case of subcritical bifurcation, there is a rapid growth of the cross-wave excitation that inevitably leads to some degree of splashing or bulging of liquid over the coated step of the container. This unwanted behaviour can be minimized by staying very close to threshold, but it cannot be eliminated entirely. The contact line is therefore not completely fixed, nor is the contact angle always exactly $90^{\circ}$. Since boundary conditions at the contact line are crucial and can contribute significantly to damping, ${ }^{35-37}$ this is likely the principal source of experimental error in the threshold values reported below. After repeated measurements, we estimate an uncertainty of $5 \%-10 \%$ in the measured values.

\section{B. Threshold of subharmonic waves}

Figure 8 (a) shows the measured threshold for cross-waves in a $9 \mathrm{~cm}$ square container using $5 \mathrm{cSt}$ silicone oil and forcing frequencies between $50 \mathrm{~Hz}$ and $75 \mathrm{~Hz}$. This is compared with the predictions of Eqs. (35) and with calculations using Dirichlet boundary conditions. As seen in the figure, the measured values are about $20 \%$ higher than those predicted by Eqs. (35) and match quite well with those obtained using Dirichlet boundary conditions. In either case, the agreement is dramatically better than it is with the boundary forced model (29), which underestimates the cross-wave threshold over this range by an order of magnitude (see Fig. 1).

Figure 8 (b) shows the measured threshold for cross-waves in a $9 \mathrm{~cm}$ square container with $10 \mathrm{cSt}$ silicone oil over the same range of forcing frequencies. These values are once more compared with the predictions of Eqs. (35) and with calculations using Dirichlet boundary conditions. The measured values are again higher than those predicted by Eqs. (35), but by a smaller amount (about 10\%) than in Fig. 8(a). The calculations with Dirichlet boundary conditions do no better in this case, overestimating the thresholds to about the same extent.

Figure 9 shows the measured threshold for cross-waves in the larger containers, which are $15 \mathrm{~cm}$ and $20 \mathrm{~cm}$ across, respectively. As with Fig. 8, a comparison is made with the predictions of Eqs. (35) and with calculations using Dirichlet boundary conditions. In these larger containers, the measured values are somewhere between the two predictions, as with the $10 \mathrm{cSt}$ experiment of Fig. 8(b). The 

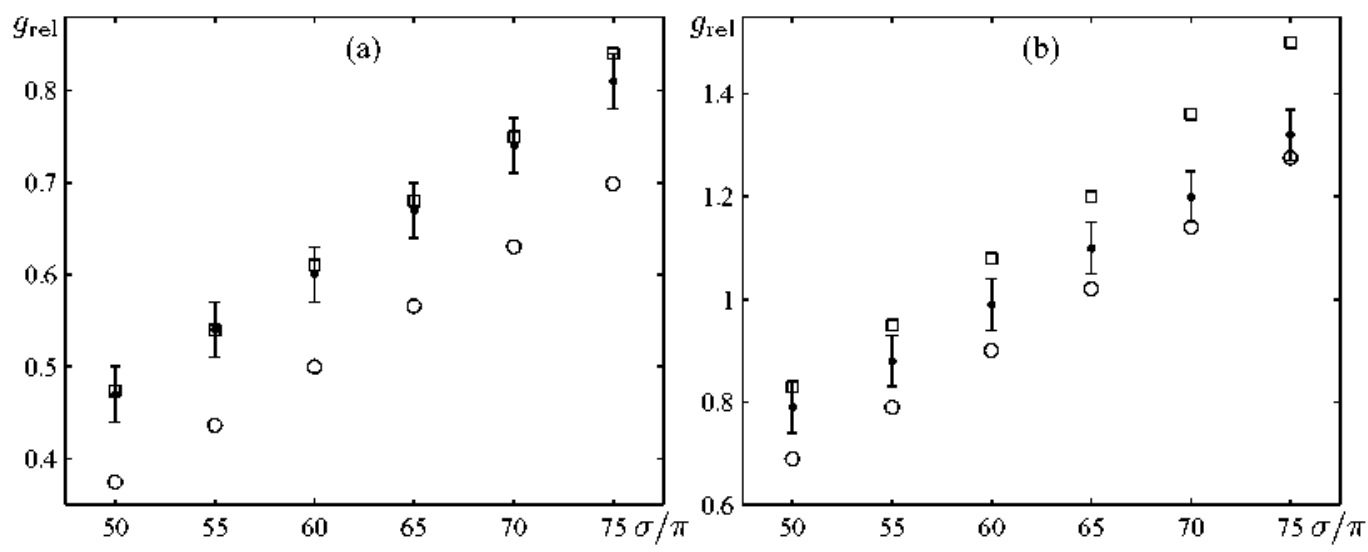

FIG. 8. Instability thresholds in a $9 \mathrm{~cm}$ siuate container with $D=5 \mathrm{~cm}$ for forcing frequencies $\sigma / \pi$ between $50 \mathrm{~Hz}$ and $75 \mathrm{~Hz}$. (a) $5 \mathrm{cSt}$ oil with $\Gamma=19.7 \mathrm{dyne} / \mathrm{cm}$. $\rho=0.913 \mathrm{~g} / \mathrm{cm}^{3}$. (b) $10 \mathrm{cSt}$ oil with $\Gamma=20.1 \mathrm{dyne} / \mathrm{cm}, \rho=0.933 \mathrm{~g} / \mathrm{cm}^{3}$. Experimentally measured thresholds (shown with estinated enror bars) are compared with the predictions of Eqs. (35) (open circles) and with Dirichlet boundary conditions instead (open squares).

difference between the measurements in the $15 \mathrm{~cm}$ container and the $20 \mathrm{~cm}$ container is very small, as is the difference between the corresponding theoretical predictions.

The main point of Figures 8 and 9 is that Eqs. (35) with distributed forcing do a much better job of matching the experimentally measured thresholds than the boundary forced model (29). As demonstrated in Fig. 1, these two models diverge dramatically for forcing frequencies beyond about $10 \mathrm{~Hz}$, with Eqs. (29) substantially underestimating the threshold in the gravity-capillary regime explored here. This difference between predicted onset values is huge (roughly a factor of 10 over the frequency range reported here) and directly due to the type of forcing balance (scaling) assumed.

An interesting secondary issue is boundary conditions. As discussed above, the boundary conditions are especially difficult to control experimentally and, consequently, it is not clear if the boundary conditions (35b) are the appropriate ones to apply. Using Dirichlet boundary conditions, instead, often gives an equally good (Figs. 8(b) and 9) or better (Fig. 8(a)) comparison with experiment. Taken together, the experiments do not clearly distinguish between the somewhat higher thresholds obtained using Dirichlet boundary conditions and those found with $\mathrm{Eq}$. (35b).
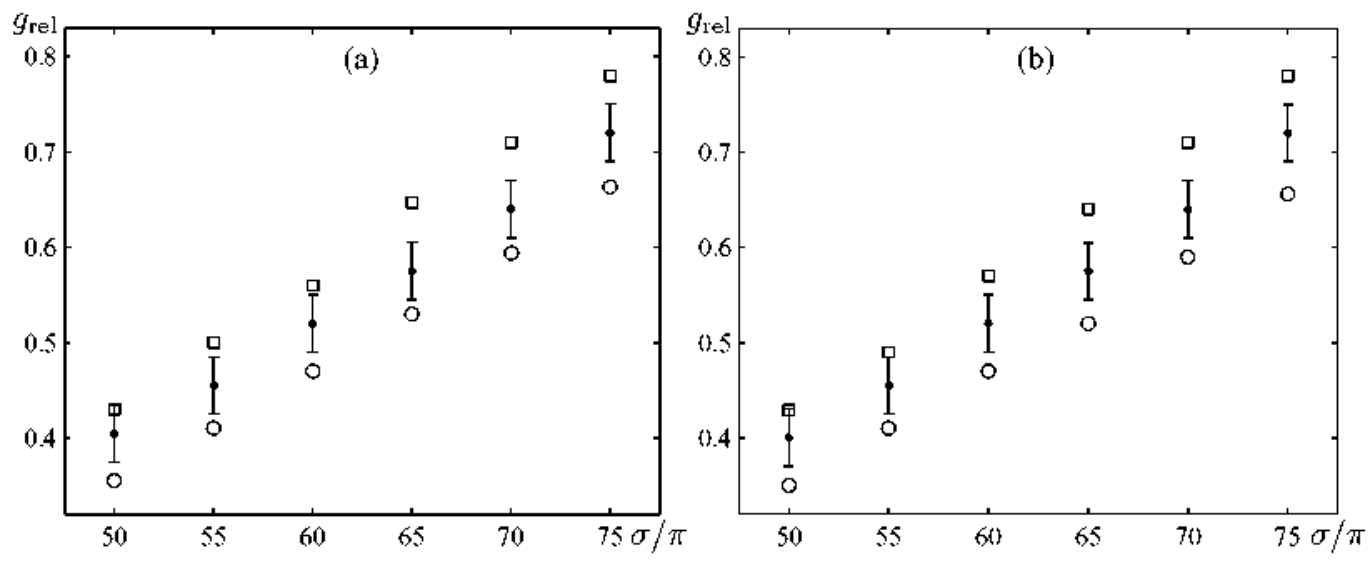

FIG. 9. Instability thresholds for $5 \mathrm{cSt}$ oil with forcing frequencies $\sigma / \pi$ between $50 \mathrm{~Hz}$ and $75 \mathrm{~Hz}$ in (a) a $15 \mathrm{~cm}$ sinare containet and (b) a $20 \mathrm{~cm}$ square container. Experimentally measured thresholds (shown with estimated error bars) are compared with the predictions of Eqs. (35) (open circles) and with Dirichlet boundary conditions instead (open squares). Remaining parameters are as in Fig. 1. 


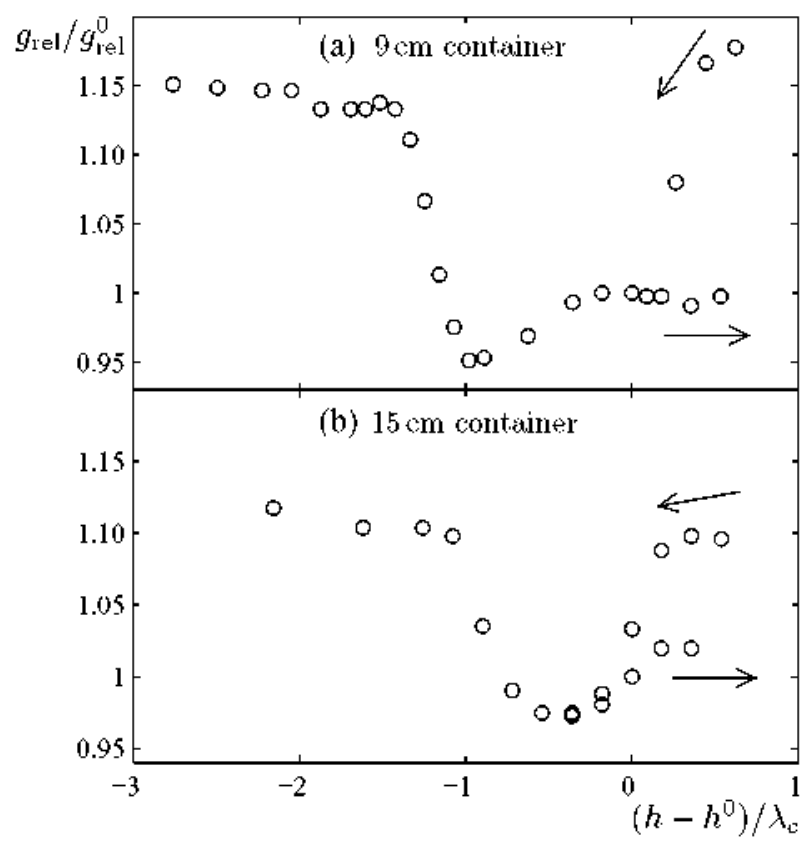

FIG. 10. Dependence of threshold on flling level for $5 \mathrm{cSt}$ oil with (a) a $9 \mathrm{~cm}$ square container, and (b) a $15 \mathrm{~cm}$ sijuate containet. The threshold is scaled by the initial value $3_{\text {rel }}^{0}$ cortesponding to a flat surface while the change in fluid depth is scaled by the capillary length $\lambda_{c}=\sqrt{(\Gamma / \rho g)} \simeq 1.48 \mathrm{~mm}$. Arrows indicate the hysteretic behaviour in the overfilled (splash prone) region.

A more quantitative idea of the influence of the boundary conditions can be obtained by investigating the dependence of the threshold on the filling level. If the container is underfilled, the effect of contact line motion increases damping and raises the threshold. If the container is overfilled, the fluid tends to splash over the step and increase damping in that way. The minimum threshold is realized by avoiding these two competing effects. Ideally, this will occur with a perfectly flat surface $\left(90^{\circ}\right.$ contact angle) but, in practice, the minimum threshold is usually observed in a slightly underfilled container, which more effectively avoids splashing.

The results of threshold measurements with varying filling levels are shown in Fig. 10. This figure shows the relative onset value (measured with respect to that of a flat surface) as a function of the change in filling level (in units of the capillary length). In the $9 \mathrm{~cm}$ square container of Fig. 10(a), the threshold first decreases as fluid is removed. As the gap between the (interior) fluid level and the step (contact line) increases toward the capillary length, the threshold decreases by about $5 \%$. After this, however, the threshold begins rapidly to rise again and, by the time the gap reaches 1.5 capillary lengths, the threshold is nearly $15 \%$ higher than its initial value. The explanation for this increase is most likely the motion of the contact line (which is no longer attached to the step). Further removal of fluid continues to raise the threshold, but at a much slower rate. If, on the other hand, fluid is added to the container, there is no immediate change in the threshold. After the increase in height reaches about 0.5 capillary lengths, however, there is a rapid and dramatic increase, evidently triggered by the onset of splashing. This transition is hysteretic. If fluid is again removed after splashing has occurred, the threshold maintains a higher value for several steps before rejoining the original curve near the initial point (flat surface).

In the $15 \mathrm{~cm}$ square container of Fig. 10(b), the same basic scenario takes place as in the smaller container, but with minor differences likely due to the absence of lateral steps. Compared to the $9 \mathrm{~cm}$ square container, the variations in onset value are somewhat smoother and less extreme; the increase in threshold with capillary motion and with splashing reaches about $10 \%$ rather than $15 \%$. Note also that the hysteresis region associated with overfilling extends beyond the initial point, suggesting that splashing during the course of an experimental run can lead to an error of $5 \%$ or more in onset values even if the fluid height is held constant. 


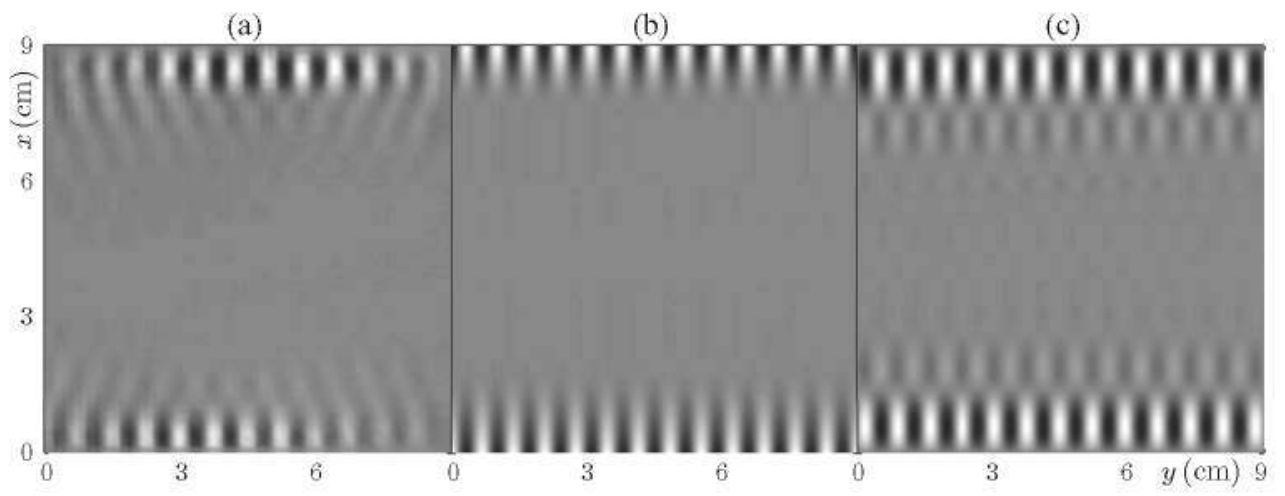

FIG. 11. (a) Experimentally observed pattern with $5 \mathrm{cSt}$ oil and $50 \mathrm{~Hz}$ forcing. (b) Corresponding eigenfunction obtained using Eqs. (35). (c) Alternative eigenfunction obtained with Dirichlet boundary conditions. Remaining parameters are as in Fig. 1.

\section{Spatial modulation}

Both the eigenfunctions predicted by Eqs. (35) and those calculated using Dirichlet boundary conditions instead of Eq. (35b) are modulated in $x$. There is, however, a greater similarity between the patterns observed experimentally and those calculated using Dirichlet boundary conditions, as seen in Fig. 11. This is because Dirichlet boundary conditions lead to a slightly faster modulation in $x$ that is more consistent with the rotated (oblique) patterns observed in the experiment, ${ }^{23}$ and because the interior part of the pattern, whose extent is needed to view the modulations clearly, is not overshadowed as much by high amplitude motion at the boundary.

A more quantitative comparison of the predicted spatial modulation with the experimentally measured profile is provided in Fig. 12 for the case of a $20 \mathrm{~cm}$ square container with $5 \mathrm{cSt}$ oil driven at $50 \mathrm{~Hz}$. The calculated curves correspond to Eqs. (35) (solid curves), Dirichlet boundary conditions in place of Eq. (35b) (dashed curves), and the boundary forced system (29) (dash-dotted curves). The amplitude and temporal phase of the theoretical solutions are matched to the experimental measurements over the region of the exponential tail. Note that all three calculations predict the modulation rate (wavenumber) reasonably well in this far-field region $(x \gtrsim 1.5)$, which reflects the
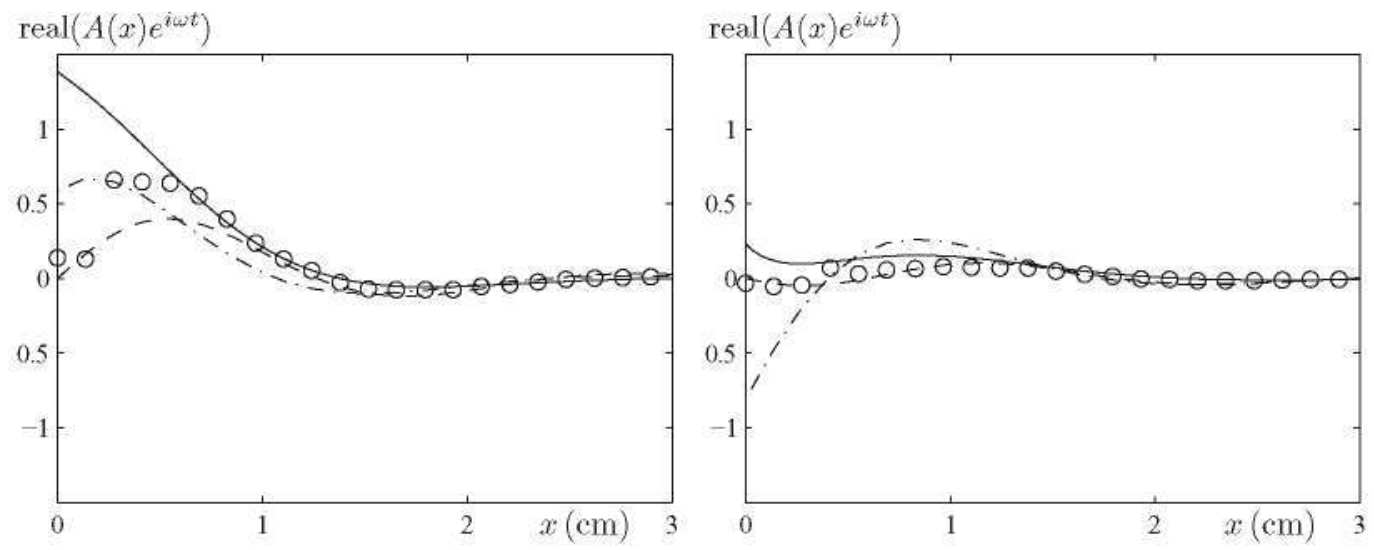

FIG. 12. Profiles (open circles) observed just above onset in an experiment in a $20 \mathrm{~cm}$ square container ( $5 \mathrm{~cm}$ deep) with $5 \mathrm{cSt}$ oil and $50 \mathrm{~Hz}$ forcing at two different temporal phases. In the left figure the waves are passing through a maximum near the endwall, while in the right figure they are passing through a minimum. These measurements are compared to the predicted profiles of Eqs. (35) (solid curve), to those calculated with Dirichlet boundary conditions instead of Eq. (35b) (dashed curve), and to those of Eqs. (29) with boundary forcing (dash-dotted curve); remaining parameters are as in Fig. 1. 


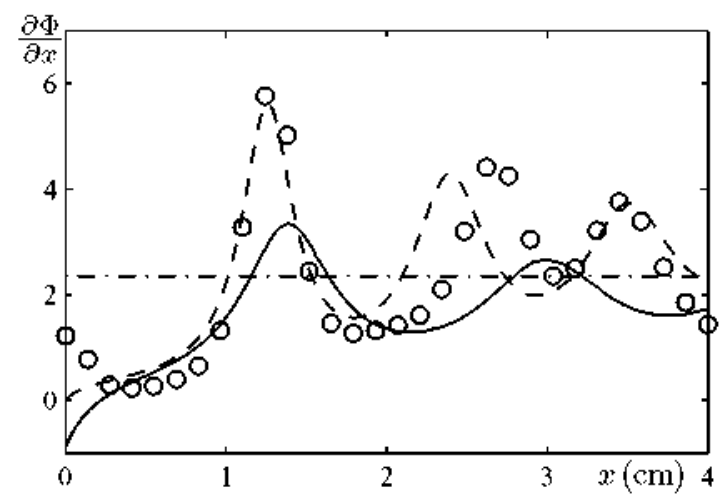

FIG. 13. The spatial detivative of the phase of $A(x), \partial \Phi(x) / \partial x$, which can be interpreted as a local wavenumber, for the same experiment as Fig. 12. Experimental results (open circles) are compared to the predictions of Eqs. (35) (solid curve). of calculations using Dirichlet boundary conditions (dashed curve), and of Eqs. (29) with boundary forcing (dashed-dotted line).

diminishing influence of the forcing. It is in the near-field (supercritical) region by the boundary where the models differ most.

The distributed forcing model (35) describes the spatial dependence of the amplitude and phase somewhat better with Dirichlet boundary conditions than with the derived boundary conditions (35b), although both do fairly well. The boundary forced model (29), in contrast, fails to capture the spatial dependence of the phase, as is evident in the right panel.

The spatial dependence of the phase, $\Phi(x)$, is a key difference between the distributed forcing model (35) and the boundary forced model (29), whose eigenfunction (in the semi-infinite case, a good approximation with these parameters) is a damped traveling wave with constant wavenumber. The inability of such a simple function to capture the spatial dependence of the phase is demonstrated in Fig. 13. In the experiment (open circles in the figure) and in Eqs. (35), whether using boundary conditions (35b) (solid curve) or Dirichlet boundary conditions (dashed curve), there are spatial variations in the derivative of $\Phi(x)$, which can be considered as a kind of local wavenumber. These variations (oscillations) cannot be captured by a single Fourier component like that predicted by Eqs. (29). The distributed forcing model (35), however, describes the measured spatial dependence of the phase $\Phi(x)$ reasonable well, especially when Dirichlet boundary conditions are used.

\section{Temporal modulation}

Modulated solutions like that shown in Fig. 6 are predicted by Eqs. (35). These quasiperiodic solutions, however, are very difficult to observed experimentally because, at the frequencies used in Sec. VI B, the waves generated at opposite sides are well-separated at onset and do not interact appreciably. As shown in Sec. VII, this means that the Hopf (modulation) frequency is very small and that the eigenfunctions can only be expected to persist (if they are stable at all) over a very narrow region of forcing values. Extreme precision and extremely long times would be needed to observe the modulation. To avoid these difficulties, we look for modulated solutions at lower frequency, where the interaction between opposing wavetrains is stronger due to the lower dimensionless damping (larger Re).

Figure 14 shows four snapshots of a modulated solution with a period of approximately $45.6 \mathrm{~s}$. It is found immediately after onset in a $20 \mathrm{~cm}$ container of $5 \mathrm{cSt}$ oil vibrated at $13.95 \mathrm{~Hz}$. Although the pattern extends far into the container and the interaction of the two sides is therefore not small, the characteristic cycling illustrated in Fig. 6, from waves concentrated on one side (a), to balanced excitation (b), to waves concentrated on the other side (c), back to balanced excitation (d), and so on, can be clearly seen.

This modulated solution persists across a range of nearby forcing frequencies and, consistent with Fig. 5(b), its period is observed to depend strongly on frequency (detuning), as shown in Fig. 15. This figure also shows the predicted Hopt frequency of Eqs. (35) with the boundary conditions (35b) 


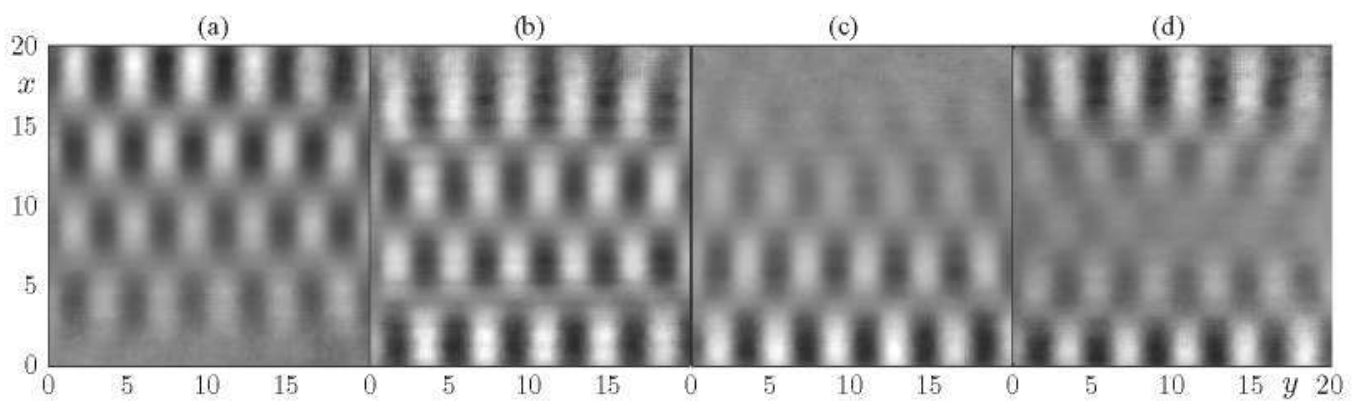

FIG. 14. Modulated (quasiperiodic) solution found in a $20 \mathrm{~cm}$ square container with $5 \mathrm{cSt}$ oil driven at $13.95 \mathrm{~Hz}$. The crosswise mode number is $N=11$ and the modulation period is about $45.6 \mathrm{~s}$. The snapshots (a)-(d) are separated by approximately $1 / 8$ of this period, or $5.7 \mathrm{~s}$.

(solid curve in the figure) and with Dirichlet boundary conditions (dashed curve). Both the order of magnitude of the modulation frequency and its oscillations with detuning are broadly consistent with the theory. There is particularly good agreement with the calculations using Dirichlet boundary conditions if a leftward shift of $0.15 \mathrm{~Hz}$ is applied to the experimental data; such a shift of effective detuning, corresponding to a $1 \%$ shift of resonance frequency, is within the level of experimental uncertainty. Figures 14 and 15 confirm one of the most interesting predictions of Eqs. (35), and other models ${ }^{28,33}$ that take account of interaction between the two opposing endwalls.

Although the results in this section seem to suggest that the experiments are closer to Dirichlet boundary conditions than those of Eq. (35b), the correct treatment of boundary conditions is a subtle and difficult problem, ${ }^{36,38}$ which is beyond the scope of this paper. As already discussed, boundary effects are the principal source of experimental error. We have focused here on extending the established NLS models to the regime of gravity-capillary waves and distributed forcing and thus showing how critical the initial scaling assumptions are. Regardless of the accuracy of the boundary conditions, the difference between boundary forcing and distributed forcing is clear and dramatic. If the depth of the endwall or wavemaker is not small compared to the extent of the cross-wave field - and in many experiments it will not be - then an extended forcing mechanism must be considered.

\section{SYMMETRY CONSIDERATIONS}

The appearance of modulated solutions in Eqs. (35) can be understood in terms of symmetry. Equations (29) and (32), for instance, possess the usual subharmonic (odd) symmetry $A \rightarrow-A$

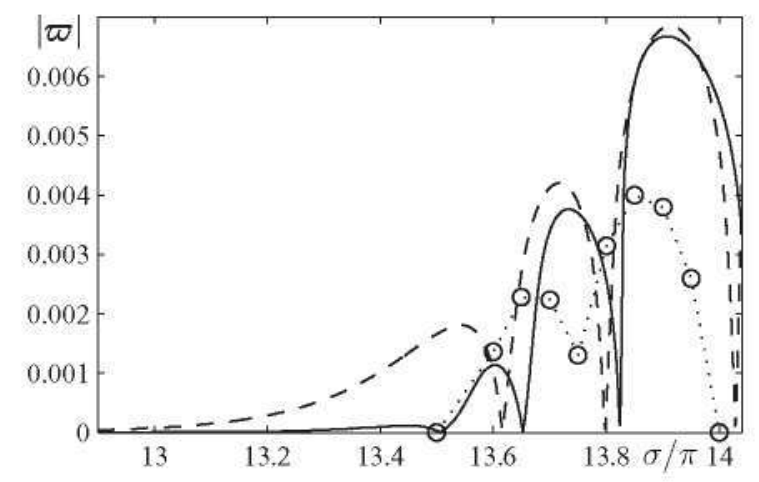

FIG. 15. Modulation (Hopf) frequency of the solution shown in Fig. 14 over an interval of forcing frequencies (in $\mathrm{Hz}$ ) around the resonance frequency $\omega_{0} / \pi=13.52 \mathrm{~Hz}$. The experimentally measured Hopf frequency $\omega$ is compared with that predicted by Eqs. (35) with the derived boundary conditions (solid curve) and with Dirichlet boundary conditions (dashed curve); remaining parameters for these calculations are as in Fig. 1. 
coming from the invariance of the original problem under time translation through a full period of the forcing; this ensures that there are only odd terms in the amplitude equation for $A$. The primary bifurcation of this parametrically forced system is steady, as found in Sec. IV, and signals the appearance of temporally unmodulated subharmonic solutions of the original problem. by

If the effects of spatial dependence are ignored for simplicity, then Eqs. (32) may be replaced

$$
i A_{t}=\left(v-i \gamma_{0}\right) A+\epsilon \bar{A}+\epsilon^{2} \beta|A|^{2} A,
$$

where it is understood that this equation applies only within a large but isolated region near the left endwall of the container. Near the right endwall of the container, where the forcing is out of phase by $\pi$, the same equation applies, but with $\epsilon \rightarrow-\epsilon$ :

$$
i B_{t}=\left(v-i \gamma_{0}\right) B-\epsilon \bar{B}+\epsilon^{2} \beta|B|^{2} B .
$$

Taken together, the equations for $A$ and $B$ exhibit the symmetry $D_{4}$, generated by $\rho:(A, B) \rightarrow i(B$, $A)$ and a reflection $R:(A, B) \rightarrow(-A, B)$; the refection $(A, B) \rightarrow(A,-B)$ is represented by $\rho^{3} R \rho$. In other words, in the absence of interaction, the phase of subharmonic waves at either end of the container, which differ by $\pm \pi / 2$, may be independently shifted by $\pi$ to generate equivalent solutions. The pitchfork bifurcation at $\epsilon^{2}=\left|\gamma_{0}\right|^{2}+v^{2}+2 v \operatorname{imag}\left(\gamma_{0}\right)$ produces eight branches $(A, B)$ of the form $\pm(A, 0), \pm(0, i A), \pm(A, i A)$, and $\pm(A,-i A)$. Assuming $\beta>0$, this pitchfork bifurcation is supercritical if $v+\operatorname{imag}\left(\gamma_{0}\right)<0$ and subcritical otherwise.

The effect of coupling between the two sides may be represented by the modified system

$$
\begin{aligned}
& i A_{t}=\left(v-i \gamma_{0}\right) A+\epsilon \bar{A}+\epsilon^{2} \beta|A|^{2} A+\mu B, \\
& i B_{t}=\left(v-i \gamma_{0}\right) B-\epsilon \bar{B}+\epsilon^{2} \beta|B|^{2} B+\mu A,
\end{aligned}
$$

where $|\mu| \ll 1$ is a small parameter if the patterns concentrated at each endwall are well separated. The value of $\mu$ will depend on the overlap of these two patterns in the interior of the container, and this is expected to oscillate as parameters (and hence the pattern wavelength) are varied, consistent with Fig. 5.

The $O(|\mu|)$ coupling terms break the $D_{4}$ symmetry of the uncoupled problem, leaving only the subgroup $C_{4}$ (cyclic group of order 4 ) generated by the rotation $\rho$. Unlike in the case of $D_{4}$, there are no nontrivial isotropy subgroups, the bifurcation problem does not separate and, in general, the critical eigenvalues are complex. The Hopf frequency associated with these complex critical eigenvalues leads to a rotation or drift through the formerly invariant subspaces defined by the $D_{4}$-symmetric problem. This drift is illustrated in Fig. 16 and confirmed by the simulations of

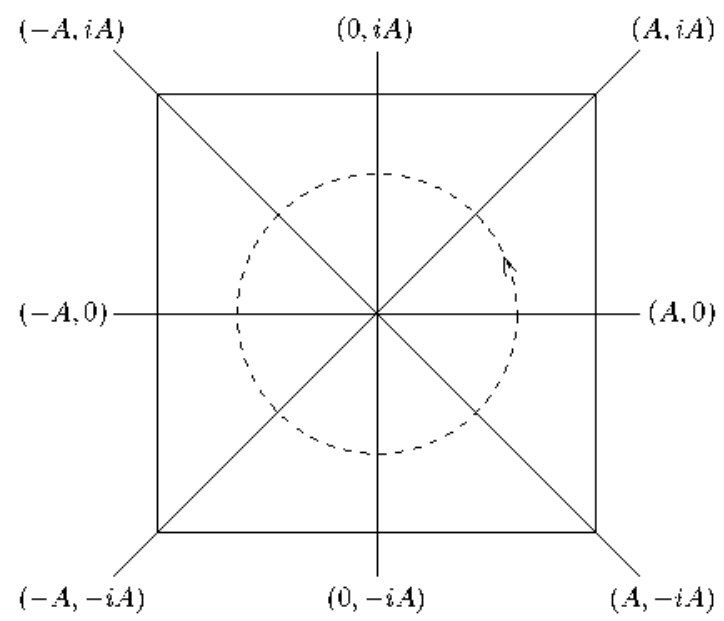

FIG. 16. Sketch showing the effect of $D_{4}$ symmetry-breaking in Eqs. (40). Modulated solutions slowly sanple each of the previously invariant subspaces. transforming repeatedly between one-sided and two-sided patterns. 
Fig. 6 and the experimental results in Fig. 14. Modulated solutions slowly alternate between onesided excitation and two-sided excitation with the (approximate) phase relationships depicted in Fig. 16.

Of course, if $|\mu|$ is small, the Hopf frequency will be small, as in Figs. 5 and 15, and the modulation period will be long. Furthermore, for $|\mu| \ll 1$, the modulated solutions arising there will not persist far from onset; beyond an $O(|\mu|)$ size interval, the solutions will again be well-described by the steady branches of the unperturbed problem. Both of these properties, the long time interval involved and the small parameter range, help explain why modulated solutions, which slowly cycle between cross-waves concentrated on the left and cross-waves concentrated on the right, are difficult to observe experimentally when the coupling is weak. In Sec. VI D, these difficulties were overcome by using a lower forcing frequency, which reduces the (dimensionless) damping so that waves penetrate further into the container. Although the interaction is not weak in that case, the above arguments still apply to an extent, and cycling behaviour like that sketched in Fig. 16 is observed in the experiment (Fig. 14).

\section{CONCLUSION}

We extended the standard theory describing weakly modulated subharmonic cross-waves in horizontally vibrated containers to include the effects of surface tension and distributed forcing, and compared the predictions of this theory with experimental results. Prior cross-wave analyses based on the work of Jones ${ }^{15}$ assumed a separation of scales between the slow spatial modulation of the cross-wave pattern and the fast spatial decay of the localized harmonic mode (oscillating bulk flow) attached to the endwalls, which provides the forcing mechanism for exciting subharmonic waves. This assumption led to the forcing appearing solely in the boundary condition for an NLS equation describing the slowly varying amplitude $A(x, t)$. While this approach is justified for cross-wave experiments where viscosity is small enough that the spatial modulation of the pattern is very slow, $18.20 .22 .24-26$ or where the wavemaker is very shallow, it does not apply to more recent experiments performed with higher frequencies ${ }^{5,7,23}$ where significant spatial modulation is observed. In the experiments reported here, and those of Porter et al. ${ }^{23}$ the oscillating bulk flow extends throughout the container and must be modeled by a distributed forcing term.

The damped NLS equation with distributed forcing derived here is a straightforward extension of the standard theoretical approach and, in particular, care was taken to follow the derivation of Bernoff $e t a l{ }^{17}$ as closely as possible. Equations (32) reduce to the model obtained in that reference if surface tension is set to zero (and the remaining nonlinear curvature term is neglected in Eq. (12c) as well) and the oscillating bulk flow is assumed to decay on a fast lengthscale compared to the modulation of $A(x, t)$.

A quantitative comparison was made between the boundary forced model (29), which is similar to others in the literature, ${ }^{15,16,18,21,27}$ and the distributed forcing model (32). Apart from the low frequency limit, these two models diverge dramatically in their predictions. The scaling with viscosity $v$ is different-critical acceleration is roughly proportional to $v$ in the case of distributed forcing and to $\sqrt{\nu}$ in the case of boundary forcing (see Fig. 2) - as are the predicted thresholds (see Fig. 1). In general, boundary forced models tend to overestimate the efficacy of the forcing since an integral over the entire oscillating bulk flow is used and this will include a region where the forcing is not strong enough to support waves. With distributed forcing, there is a natural distinction between supercritical regions, where the forcing is sufficient to locally excite subharmonic waves and underforced subcritical regions, where the waves lose energy and decay.

An interesting feature of distributed forcing models is the multiplicity of cross-waves solutions that may be excited with the same crosswise mode number $N$. These secondary solutions are distinguished by the number of spatial oscillations they undergo in the supercritical region and cannot be found in boundary forced models. The importance of these secondary modes increases with the width of the forcing mechanism (the depth of the container or wavemaker).

Boundary conditions at the endwall (wavemaker) play a crucial role, both in theory and experiment, ${ }^{23}$ affecting not only the minimum onset value but the leftward or rightward shift of the neutral stability curve (i.e., the detuning value at this minimum). The influence of boundary 
conditions, which are the principal source of uncertainty for the experiments presented here, can be quantitatively seen in Fig. 10. Because the distributed forcing term appears directly in the amplitude equation, alternative boundary conditions can be sensibly applied without disrupting the essential balance between forcing and damping. In fact, simple Dirichlet boundary conditions were seen to work quite well, leading both to a more accurate prediction of threshold acceleration in some cases (see Fig. 8(a)) and to a more rapidly modulated pattern consistent with experiment (see Figs. 11-13).

Finally, it was seen how horizontal excitation in a finite-sized container, with two opposing out-of-phase wavetrains, naturally leads to a Hopf bifurcation and thus to temporally quasiperiodic eigenfunctions that cycle slowly between two-sided and one-sided patterns. ${ }^{28,33}$ If the overlap of the subharmonic waves originating at opposite endwalls is small, this can be understood as the result of a symmetry-breaking interaction that destroys the $D_{4}$ symmetry of the uncoupled (infinite length) problem. The small interaction term leads to a similarly small Hopf frequency and a small interval of forcing values where quasiperiodic solutions can be found. In Sec. VI D, the interaction strength was increased by using lower forcing frequencies, near $13.5 \mathrm{~Hz}$, allowing the existence of quasiperiodic solutions to be experimentally confirmed. The slow modulation frequency was consistent with the prediction of Eqs. (35).

The results presented here demonstrate the important qualitative and quantitative consequences of assuming a separation of lengthscales between the forcing mechanism and the cross-wave modulation. Avoiding such an assumption and the resulting boundary forcing term is essential for any theory aiming to capture the thresholds and features of the more complicated patterns observed in the gravity-capillary regime. ${ }^{5,7,23}$ When compared with boundary forced models such as Eqs. (29), the success of the distributed forcing model (35) in capturing the experimentally measured thresholds in the gravity-capillary regime, the observed spatial modulation of onset patterns, and the slow temporal modulation observed with lower frequencies, is considerable. The distributed forcing model (35) describes the experiments reported here quite well, and many of those in Porter et al. ${ }^{23}$ despite the significant spatial modulation (rotation) of the patterns. It should be remembered, though, that Eqs. (35) were derived with the assumption of (weakly) modulated cross-waves and thus cannot describe subharmonic patterns that depart too much from a crosswise orientation. An alternative framework that permits more general oblique patterns is considered elsewhere. ${ }^{28}$

\section{ACKNOWLEDGMENTS}

This work was supported by the Spanish Ministry of Science and Innovation (currently, Ministry of Economy and Competitiveness) under project AYA2010-19081. We acknowledge helpful conversations about cross-waves with José M. Vega and Fernando Varas.

APPENDIX: DETAILS OF THE DERIVATION IN SEC. III

\section{Second and third order solutions}

At second order

$$
\Delta \phi^{(2)}=-2\left(\phi_{x X}^{(1)}+\phi_{y Y}^{(1)}\right),
$$

with boundary conditions at the surface $z=0$

$$
\begin{aligned}
& f_{t}^{(2)}-\phi_{z}^{(2)}=-f_{T_{1}}^{(1)}+f^{(1)} \phi_{z z}^{(1)}-\bar{\nabla} \phi^{(1)} \cdot \bar{\nabla} f^{(1)}, \\
& \phi_{t}^{(2)}+G_{0} f^{(2)}-\Gamma_{0} \underline{\Delta} f^{(2)}=-\phi_{T_{2}}^{(1)}-\frac{1}{2}\left|\nabla \phi^{(1)}\right|^{2}+2 \Gamma_{0}\left(f_{x X}^{(1)}+f_{y Y}^{(1)}\right)-f^{(1)} \phi_{z t}^{(1)},
\end{aligned}
$$

and at the boundaries

$$
\begin{array}{r}
\hat{\phi}_{x}^{(2)}=-\hat{\phi}_{X}^{(1)}-\frac{\delta}{\sqrt{i \Omega}} \hat{\phi}_{x x}^{(1)} \quad \text { at } \quad x=0, \\
\phi^{(2)} \rightarrow 0 \text { as } \quad x \rightarrow \infty,
\end{array}
$$




$$
\begin{array}{ll}
\hat{\phi}_{y}^{(2)}=-\hat{\phi}_{Y}^{(1)} \mp \frac{\delta}{\sqrt{i \Omega}} \hat{\phi}_{y y}^{(1)} & \text { at } \quad y=0, N \pi, \\
\hat{\phi}_{z}^{(2)}=-\frac{\delta}{\sqrt{i \Omega}} \hat{\phi}_{z z}^{(1)} & \text { at } \quad z=-d .
\end{array}
$$

At third order, keeping only the dispersive terms that are required to determine onset, we have

$$
\Delta \phi^{(3)}=-\phi_{X X}^{(1)}
$$

with boundary conditions at the surface $z=0$

$$
\begin{aligned}
& f_{t}^{(3)}-\phi_{\bar{c}}^{(3)}=2 \delta \Delta f^{(1)}-f_{T_{2}}^{(1)}-v^{(2)} f^{(1)}, \\
& \phi_{t}^{(3)}+G_{0} f^{(3)}-\Gamma_{0} \Delta f^{(3)}=-\phi_{T_{2}}+f_{X X}^{(1)}-2 \delta \phi_{\bar{\alpha}}^{(1)}-v^{(2)} \phi^{(1)},
\end{aligned}
$$

and at the boundaries

$$
\begin{array}{lll}
\phi_{x}^{(3)}=0 & \text { at } & x=0, \\
\phi^{(3)} \rightarrow 0 & \text { as } & x \rightarrow \infty, \\
\phi_{y}^{(3)}=0 & \text { at } & y=0, N \pi, \\
\phi_{z}^{(3)}=0 & \text { at } & z=-d .
\end{array}
$$

Additional terms contributing to the cubic self-interaction coefficient are considered separately in Sec. 3 of this Appendix.

Dissipation at the walls is taken into account ${ }^{17}$ by including a localized velocity contribution,

$$
\hat{\phi}_{z} \rightarrow \hat{\phi}_{z}\left(1-\sum_{\text {edges }} e^{-\sqrt{t \Omega \operatorname{Re}} \xi_{1}}\right)
$$

in the free surface condition. Here $\xi_{i}$ denotes the perpendicular distance from the wall in question. Note that, although this exponential contribution is formally $O(1)$ it contributes only over an $O(\epsilon)$ region near each wall and is thus taken to be of that order. ${ }^{17}$

\section{Solvability condition}

The solvability condition for Eqs. (A1) and (A2) is obtained from Green's identity:

$$
\begin{aligned}
0 & =\int_{D} \Delta \phi^{\dagger} \phi^{(i)} d x d y d z d t=\int_{D} \phi^{\dagger} \Delta \phi^{(i)} d x d y d z d t+\int_{x=0} \phi^{\dagger} \phi_{x}^{(i)} d y d z d t+\int_{y=0} \phi^{\dagger} \phi_{y}^{(i)} d x d z d t \\
& -\int_{y=N \pi} \phi^{\dagger} \phi_{y}^{(i)} d x d z d t+\int_{\bar{z}=-d} \phi^{\dagger} \phi_{z}^{(i)} d x d y d t-\int_{z=0}\left(\phi^{\dagger} \phi_{z}^{(i)}-\phi_{z}^{\dagger} \phi^{(i)}\right) d x d y d t
\end{aligned}
$$

where $D$ denotes the entire fluid domain and the surface integrals are taken over the specified boundaries. Note the integral over time, which will be used later to select the subharmonic component.

In order to extract terms with subharmonic time dependence, we take $\left(\phi^{\dagger}, f^{\dagger}\right)$ as solutions of the homogeneous (unforced) version of Eqs. (17) (which defines a self-adjoint operator for the standard 
scalar product) oscillating as $\exp \{-i t\}$. The final term in Eq. (A4) can be rewritten as

$$
\begin{aligned}
& \int_{z=0}\left(\phi^{\dagger} \phi_{z}^{(i)}-\phi_{z}^{\dagger} \phi^{(i)}\right) d x d y d t=\int_{z=0}\left[\phi^{\dagger}\left(\phi_{z}^{(i)}-f_{t}^{(i)}\right)+\phi^{\dagger} f_{t}^{(i)}-f_{t}^{\dagger} \phi^{(i)}\right] d x d y d t \\
& =\int_{z=0}\left[\phi^{\dagger}\left(\phi_{\bar{c}}^{(i)}-f_{t}^{(i)}\right)+f^{\dagger}\left(\phi_{t}^{(i)}+G_{0} f^{(i)}-\Gamma_{0} \underline{\Delta} f^{(i)}\right)\right] d x d y d t \\
& +\Gamma_{y=N \pi, \bar{c}=\dot{y}} f_{y}^{\dagger} f_{y}^{(i)} d x d t-\Gamma_{0} \int_{y=\bar{\varepsilon}=0} f^{\dagger} f_{y}^{(i)} d x d t+\Gamma_{0} \int\left(f_{x}^{\dagger} f^{(i)}-f^{\dagger} f_{x}^{(i)}\right) d y d t,
\end{aligned}
$$

where periodicity has been used in integrating by parts in time. Finally, the term in Eq. (A5) with the unknown $f_{x}(0, y)$ is rewritten as

$$
\Gamma_{0} \int_{x=z=0}\left(f_{x}^{\dagger} f^{(i)}-f^{\dagger} f_{x}^{(i)}\right) d y d t=i \Gamma_{0} \int\left[f^{\dagger}\left(f_{x t}^{(i)}-\phi_{x z}^{(i)}\right)+f^{\dagger} \phi_{x z}^{(i)}\right] d y d t .
$$

Here, assuming continuity, $f_{x t}^{(i)}-\phi_{x z}^{(i)}$ is evaluated by approaching the contact line from the free surface and $\phi_{x z}^{(i)}$ by approaching along the wall. Combining these expressions yields the solvability condition

$$
\begin{aligned}
& 0=\int_{D} \phi^{\dagger} \Delta \phi^{(i)} d x d y d z d t+\int_{x=0} \phi^{\dagger} \phi_{x}^{(i)} d y d z d t+\int_{y=0} \phi^{\dagger} \phi_{y}^{(i)} d x d z d t-\int_{y=N \pi} \phi^{\dagger} \phi_{y}^{(i)} d x d z d t \\
& +\int_{z=-d^{\prime}} \phi^{\dagger} \phi_{z}^{(i)} d x d y d t+\int_{z=\dot{y}} \phi^{\dagger}\left(f_{t}^{(i)}-\phi_{z}^{(i)}\right)-f^{\dagger}\left(\phi_{t}^{(i)}+G_{0} f^{(i)}-\Gamma_{0} \Delta f^{(i)}\right) d x d y d t \\
& -\Gamma_{0} \int_{y=N \pi, z=0} f^{\dagger} f_{y}^{(i)} d x d t+\Gamma_{0} \int_{y=z=0} f^{\dagger} f_{y}^{(i)} d x d t+i \Gamma_{0} \int_{x=z=0} f^{\dagger}\left(f_{x t}^{(i)}-\phi_{x z}^{(i)}\right)+f^{\dagger} \phi_{x z}^{(i)} d y d t .
\end{aligned}
$$

\section{Self-interaction coefficient}

The relevant terms appearing in the free surface boundary conditions (12b) and (12c) are

$$
\begin{gathered}
f_{t}^{(3)}-\phi_{z}^{(3)}=f^{(2)} \phi_{z z}^{(1)}+f^{(1)} \phi_{z z}^{(2)}-\bar{\nabla} \phi^{(2)} \cdot \bar{\nabla} f^{(1)}-\bar{\nabla} \phi^{(1)} \cdot \bar{\nabla} f^{(2)} \\
+\frac{1}{2} \phi_{z z \bar{z}}^{(1)} f^{(1)} f^{(1)}-f^{(1)} \bar{\nabla} \phi_{\bar{z}}^{(1)} \cdot \bar{\nabla} f^{(1)}, \\
\phi_{t}^{(3)}+H f^{(3)}-\Gamma_{0} \underline{\Delta} f^{(3)}=-\nabla \phi^{(1)} \cdot \nabla \phi^{(2)}-f^{(2)} \phi_{z t}^{(1)}-f^{(1)} \phi_{z t}^{(2)}-\frac{1}{2} \phi_{z z t}^{(1)} f^{(1)} f^{(1)} \\
-f^{(1)} \nabla \phi_{z}^{(1)} \cdot \nabla \phi^{(1)}+\frac{1}{2} G_{0} f^{(1)}\left|\bar{\nabla} f^{(1)}\right|^{2}-\frac{1}{2} \Gamma_{0} \bar{\nabla} \cdot\left[\bar{\nabla} f^{(1)}\left|\bar{\nabla} f^{(1)}\right|^{2}\right],
\end{gathered}
$$

where $\phi^{(2)}$ and $f^{(2)}$ are the nonresonant parts of the solution to Eqs. (AL):

$$
\begin{aligned}
& \phi^{(2)}=\frac{1}{8}\left(i A^{2} e^{2 i t}+c . c .\right)\left\{1+3 H^{-2}+3 \frac{\left(1+H^{-2}\right)\left(H-H^{-1}+2 \Gamma_{i}\right)}{3 \Gamma_{0}-H^{-1}} \cos (2 y) \frac{\cosh [2(z+d)]}{\cosh (2 d)}\right\}, \\
& f^{(2)}=\frac{1}{4}\left(A^{2} e^{2 i t}+c . c\right) \frac{3-H^{-2}}{3 \Gamma_{0}-H^{-1}} \cos (2 y)+\frac{1}{2}|A|^{2}\left[\frac{H^{-2}-1}{H-\Gamma_{0}}+\frac{H^{-2}+1}{H+3 \Gamma_{0}} \cos (2 y)\right] . \quad \text { (A } 10
\end{aligned}
$$




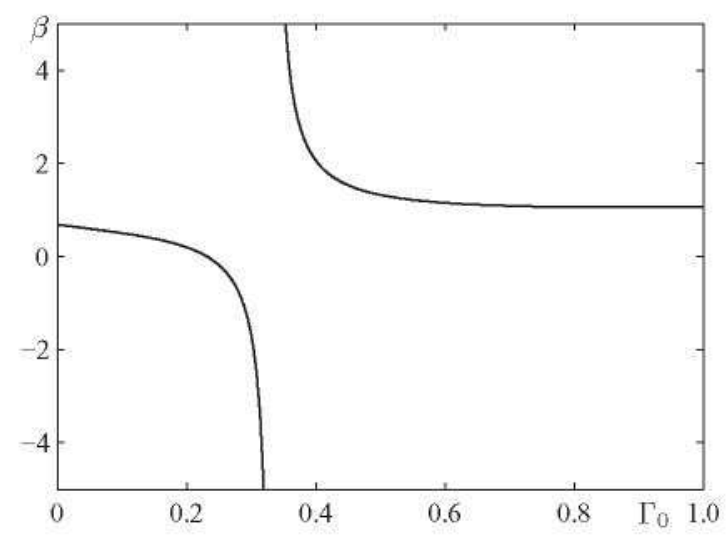

FIG. 17. Cubic coefficient $\beta$ as a function of $\Gamma_{0}$ for large depth $(H=1)$.

Applying the solvability condition (A7), the self-interaction coefficient is obtained

$$
\begin{gathered}
\beta=\frac{3}{16} H^{-2}\left(2 H^{-1} \Gamma_{0}+1\right)+\frac{12 H \Gamma_{0}+H^{-2}-10+9 H^{2}}{16\left(3 H \Gamma_{0}-1\right)} \\
+\frac{2 H^{-2}-3 H^{-4}-3-\left(5 H^{-1}-14 H^{-3}+5 H^{-5}\right) \Gamma_{0}}{8\left(3 H^{-1} \Gamma_{0}+1\right)\left(H^{-1} \Gamma_{0}-1\right)} .
\end{gathered}
$$

The first term in Eqs. (A11) comes from the final two terms in Eq. (12c), which are cubic corrections due to curvature not included in Bernoff et al.,${ }^{17}$ while the second and third terms come from harmonic and mean (zero frequency) components of the second order solutions in Eq. (A10). The sum of these final two terms evaluated at $\Gamma_{0}=0$ gives the cubic coefficient $\beta$ in Eqs. (1).

There is a singularity in Eq. (A11) on the allowed interval $0 \leq \Gamma_{0} \leq 1$ when $\Gamma_{0}=H / 3$, which corresponds to the $1: 2$ resonance when the wavenumber of harmonic waves, as defined by the inviscid dispersion relation used in Eq. (4) with frequency $2 \sigma$, is exactly $2 k_{y}$. This leads to a small region of negative $\beta$ values, for $0.232 \lesssim \Gamma_{0} \leq 1 / 3$ in the limit of large depth $(H=1)$, while outside of this $\beta$ is positive, as illustrated in Fig. 17. This singularity could be removed by a more careful treatment near the 1:2 resonance including damping effects for the harmonic mode.

${ }^{1}$ M. Faraday, "On a peculiar class of acoustical figures and on certain forms assumed by groups of particles upon vibrating elastic surfaces," Philos. Trans. R. Soc. London 121, 299-340 (1831).

${ }^{2}$ M. Schuler, "Der umschlag von oberflächenwellen," Z. Angew. Math. Mech. 13, 443-446 (1933).

${ }^{3}$ M. Tatsuno, S. Inoue, and J. Okabe, "Transfiguration of surface waves," Rep. Res. Inst. Appl. Mech. (Kyushu Univ.) 17, 195-215 (1969).

${ }^{4}$ J. M. Becker and J. W. Miles, "Standing radial cross-waves," J. Fluid Mech. 222, 471-499 (1991).

${ }^{5}$ S. Taneda, "Visual observations of the flow around a half-submerged oscillating circular cylinder," Fluid Dyn. Res. 13, 119-151 (1994).

${ }^{6}$ T. S. Krasnopolskaya and G. J. F. V. Heijst, "Wave pattern formation in a fluid annulus with a radially vibrating inner cylinder," J. Fluid Mech. 328, 229-252 (1996).

${ }^{7}$ F. Moisy, G.-J. Michon, M. Rabaud, and E. Sultan, "Cross-waves induced by the vertical oscillation of a fully immersed vertical plate," Phys. Fluids 24, 022110 (2012).

${ }^{8}$ W. Zhang and J. Viñals, "Pattern formation in weakly damped parametric surface waves," J. Fluid Mech. 336, 301-330 (1997).

${ }^{9} \mathrm{~W}$. Zhang and J. Viñals, "Pattern formation in weakly damped parametric surface waves driven by two frequency components," J. Fluid Mech. 341, 225-244 (1997).

${ }^{10} \mathrm{H}$. Arbell and J. Fineberg, "Pattern formation in two-frequency forced parametric waves," Phys. Rev. E 65, 036224 (2002).

${ }^{11}$ J. Porter, C. M. Topaz, and M. Silber, "Pattern control via multifrequency parametric forcing," Phys. Rev. Lett. 93, 034502 (2004).

${ }^{12}$ A. C. Skeldon and J. Porter, "Scaling properties of weakly nonlinear coefficients in the Faraday problem," Phys. Rev. E 84, 016209 (2011).

${ }^{13}$ C. J. R. Garrett, “On Cross-waves,” J. Fluid Mech. 41, 837-849 (1970).

${ }^{14}$ J. D. Lin and L. N. Howard, "Non-linear standing waves in a rectangular tank due to forced oscillation," Hydrodynamics Laboratory Technical Report No. 44, MIT, 1960. 
${ }^{15}$ A. F. Jones, "The generation of cross-waves in a long deep channel by parametric resonance," J. Fluid Mech. 138, 53-74 (1984).

${ }^{16} \mathrm{~S}$. Lichter and J. Chen, "Subharmonic resonance of nonlinear cross-waves," J. Fluid Mech. 183, 451-465 (1987).

${ }^{17}$ A. J. Bernoff, L. P. Kwok, and S. Lichter, "Viscous cross-waves: An analytical treatment," Phys. Fluids A 1, 678-688 (1989).

${ }^{18} \mathrm{~L}$. Shemer and E. Kit, "Long-time evolution and regions of existence of parametrically excited nonlinear cross-waves in a tank," J. Fluid Mech. 209, 249-263 (1989).

${ }^{19}$ H. Ayanle, A. J. Bernoff, and S. Lichter, "Spanwise modal competition in cross-waves," Physica D 43, 87-104 (1990).

${ }^{20}$ S. Lichter and A. J. Bernoff, "Stability of steady cross waves: Theory and experiment," Phys. Rev. A 37, 1663-1667 (1988).

${ }^{21}$ E. Kit and L. Shemer, "On the neutral stability of cross-waves," Phys. Fluids A 1, 1128-1132 (1989).

${ }^{22}$ W. B. Underhill, S. Lichter, and A. J. Bernoff, "Modulated, frequency-locked, and chaotic cross-waves," J. Fluid Mech. 225, 371-394 (1991).

${ }^{23}$ J. Porter, I. Tinao, A. Laverón-Simavilla, and C. A. Lopez, "Pattern selection in a horizontally vibrated container," Fluid Dyn. Res. 44, 065501 (2012).

${ }^{24}$ B. J. S. Barnard and W. G. Pritchard, "Cross-waves. Part 2. Experiments," J. Fluid Mech. 55, 245-255 (1972).

${ }^{25}$ S. Lichter and W. B. Underhill, "Mode-number shifting of nonlinear cross-waves," Phys. Rev. A 35, 5282-5284 (1987).

${ }^{26} \mathrm{~L}$. Shemer and S. Lichter, "Identification of cross-wave regimes in the vicinity of a cut-off frequency," Phys. Fluids $\mathbf{3 0}$, 3427-3433 (1987).

${ }^{27}$ J. W. Miles, "Note on a parametrically excited, trapped cross-wave," J. Fluid Mech. 151, 391-394 (1985).

${ }^{28}$ J. M. Perez-Gracia, J. Porter, F. Varas, and J. M. Vega, "Subharmonic capillary-gravity waves in large containers subject to horizontal vibrations," J. Fluid Mech. 739, 196-228 (2014).

${ }^{29} \mathrm{~F}$. Varas and J. M. Vega, "Modulated surface waves in large-aspect-ratio horizonally vibrated containers," J. Fluid Mech. 579, 271-304 (2007).

${ }^{30}$ L. Shemer and S. Lichter, "The mode number dependence of neutral stability of cross-waves," Exp. Fluids 9, 148-152 (1990).

${ }^{31}$ J. A. Nicolás and J. M. Vega, "Three-dimensional streaming flows driven by oscillatory boundary layers," Fluid Dyn. Res. 32, 119-139 (2003).

32 T. Havelock, "LIX: Forced surface-waves on water," Philos. Mag. Ser. 7 8, 569-576 (1929).

${ }^{33}$ J. Porter, I. Tinao, A. Laverón-Simavilla, and J. Rodríguez, "Onset patterns in a simple model of localized parametric forcing," Phys. Rev. E 88, 042913 (2013).

${ }^{34}$ F. Moisy, M. Rabaud, and K. Salsac, "A synthetic Schlieren method for the measurement of the topography of a liquid interface," Exp. Fluids 46, 1021-1036 (2009).

${ }^{35}$ C. C. Mei and L. F. Liu, "The Damping of surface gravity waves in a bounded liquid," J. Fluid Mech. 59, 239-256 (1973).

${ }^{36}$ L. M. Hocking, "The damping of capillary-gravity waves at a rigid boundary," J. Fluid Mech. 179, 253-266 (1987).

${ }^{37}$ M. Perlin and W. W. Schultz, "Capillary effects on surface waves," Annu. Rev. Fluid Mech. 32, 241-274 (2000).

${ }^{38}$ M. Perlin, W. W. Schultz, and Z. Liu, "High Reynolds number oscillating contact lines," Wave Motion 40, 41-56 (2004). 